\title{
Implicit Total Variation Diminishing (TVD) Schemes for Steady-State Calculations
}

H. C. Yee, R. F. Warming, and A. Harten

March 1983 


\section{Implicit Total Variation Diminishing (TVD) Schemes for Steady-State Calculations}

H. C. Yee

R. F. Warming, Ames Research Center, Moffett Field, California

A. Harten, Tel Aviv University, Tel Aviv, Israel 


\section{ERRATA}

NASA Technical Memorandum 84342

\section{Implicit Total Variation Diminishing (TVD) Schemes for Steady-State Calculations}

H.C. Yee, R.F. Warming, and A. Harten

March 1983

Page 5, line 6: The word "notation" should be "notion".

Page 6: Equation (2.7) should be

$$
\bar{f}_{j+1 / 2}=\frac{1}{2}\left[f_{j}+f_{j+1}-\frac{1}{\lambda} \Delta_{j+1 / 2} u\right]
$$

Page 6, line 15: The line should be "where $Q\left(a_{j+1 / 2}\right)=\frac{1}{\lambda}$ ".

Page 7, line 9: The line should be "Applying condition (2.11) and/or (2.10)...".

Page 14: Equation (2.28) should be

$$
\sigma(z)=\frac{1}{2} Q(z)+\lambda(\eta-1 / 2) z^{2}
$$

Page 15: Equation (2.30) should be

$$
g=\Delta x \sigma(a) \frac{\partial u}{\partial x}+O\left((\Delta x)^{2}\right)
$$

Page 16, line 28: "The expression $q=\gamma=0$ " should be " $g=\gamma=0$ ".

Page 17: Equation (2.35b)-(2.35d) should be

$$
E_{1}=\ldots, \quad E_{2}=\ldots, \quad E_{3}=\ldots
$$

Page 21: Equation (3.7f) should be

$$
\theta_{j}^{\prime}=\frac{\left|\alpha_{j+1 / 2}^{l}-\alpha_{j-1 / 2}^{l}\right|}{\left|\alpha_{j+1 / 2}^{l}\right|+\left|\alpha_{j-1 / 2}^{l}\right|}
$$

Page 23, line 2: The first word "The" should be "A".

Page 35: Equation (5.13) should be

$$
U_{j, k}^{n+1}=\mathcal{L}_{x}^{*} L_{y}^{*} L_{y}^{*} L_{x}^{*} U_{j, k}^{n}
$$

Page 43 line 15: The word "operators" should be "operated". 


\title{
Implicit Total Variation Diminishing (TVD) Schemes for Steady-State Calculations
}

\author{
H.C. Yeet and R.F. W arming $\uparrow$ \\ NASA Ames Research Center, Moffett Field, California
}

\section{A. Harten}

Tel-Aviv University, Tel Aviv and New York University, New York

\begin{abstract}
We examine the application of a new implicit unconditionallystable high-resolution TVD scheme to steady-state calculations. It is a member of a one-parameter family of explicit and implicit second-order accurate schemes developed by Harten for the computation of weak solutions of one-dimensional hy perbolic conservation laws. This scheme is guaranteed not to generate spurious oscillations for a nonlinear scalar equation and a constant coefficient system. Numerical experiments show that this scheme not only has a fairly rapid convergence rate, but also generates a highlyresolved approximation to the steady-state solution. A detailed implementation of the implicit scheme for the one- and two-dimensional compressible inviscid equations of gas dynamics is presented. Some numerical computations of one- and two-dimensional fluid flows containing shocks demonstrate the efficiency and accuracy of this new scheme.
\end{abstract}

\section{$\S 1$. Introduction}

Conventional schemes for the solution of nonlinear hyperbolic conservation laws are linear and $L_{2}$-stable when considered in the constant coefficient case [1]. There are three major difficulties in using such schemes to compute discontinuous solutions of a nonlinear system, such as the compressible Euler equations:

(i) Schemes that are second (or higher) order accurate may produce oscillations wherever the solution is not smooth.

(ii) Nonlinear instabilities may develop in spite of the $L_{2}$-stability in the constant coefficient case.

†Research Scientist, Computational Fluid Dynamics Branch. $\Varangle$ Associate Professor, School of Mathematical Sciences. 
(iii) The scheme may select a nonphysical solution.

It is well known that monotone conservative difference schemes always converge and that their limit is the physical weak solution satisfying an entropy inequality. Thus monotone schemes are guaranteed to not have difficulties (ii) and (iii) above. However, monotone schemes are only first-order accurate. Consequently, they produce rather crude approximations whenever the solution varies strongly in space or time.

When using a second (or higher) order accurate scheme, some of these difficulties can be overcome by adding a hefty amount of numerical dissipation to the scheme. Unfortunately this process brings about an irretrievable loss of information that exhibits itself in degraded accuracy and smeared discontinuities. Thus, a typical complaint about conventional schemes which are developed under the guidelines of linear theory is that they are not robust and/or not accurate enough.

To overcome the above difficulties, we consider a new class of schemes that is more appropriate for the computation of weak solutions (i.e., solutions with shocks and contact discontinuties) of nonlinear hyperbolic conservation laws. These schemes are required (a) to be total variation diminishing in the nonlinear scalar case and the constant coefficient system case (see[2,3]), and (b) to be consistent with the conservation law and an entropy inequality [4-6]. The first property guarantees that the scheme does not generate spurious oscillations. We refer to schemes with this property as $\underline{T}$ otal $V$ ariation Diminishing (TVD) schemes (or total variation $\underline{n}$ on $\underline{\underline{z}}$ ncreasing - TVNI [2]). The latter property guarantees that the weak solutions are physical ones. The motivation for considering this class of schemes lies in the convergence theorem that states: "If a finite difference scheme is consistent with the conservation law and its entropy inequality, and the scheme is stable in the sense of uniformly bounded total variation in $x$, then the scheme is convergent and its limit is the physical weak solution of the initial value problem" (see [3]). As a result of this theorem, schemes in this class are guaranteed to avoid difficulties (i) to (iii) mentioned above.

The class of TVD schemes contains that of monotone schemes, but is signiflcantly larger, as it includes also second-order accurate schemes. Existence of second-order accurate $T V D$ schemes was demonstrated in $[2,3,7-$ 9]. Unlike monotone schemes, $T V D$ schemes are not automatically consis- 
tent with the entropy inequality. Consequently, some mechanism may have to be explicitly added to a TVD scheme in order to enforce the selection of the physical solution. In [2] and [10], Harten and Harten and Hyman demonstrate a way of modifying a TVD scheme to be consistent with an entropy inequality.

Second-order accurate TVD schemes are genuinely nonlinear in the sense that the schemes are necessarily nonlinear even in the constant coefficient case. Consequently, nonstandard design principles are needed for the construction of second-order accurate TVD schemes. In [2], Harten develops a rather general technique for converting a first-order accurate explicit $T V D$ scheme into a second-order accurate one. The second-order accuracy is obtained by applying the first-order accurate TV.D scheme to an appropriately modified flux; the so modified scheme remains TVD. This is due to the fact that the TVD property is independent of the particular form of the flux as long as it has bounded modified characteristic speeds.

In $[11,12]$, we have examined the application of an explicit second-order accurate $T V D$ scheme [2] to steady-state calculations. Numerical experiments show that this explicit scheme generates nonoscillatory, highly accurate steady-state solutions.

The time-consistent approach to steady-state may be thought of as the temporal decay of the initial error. The rate of decay associated with the pure initial value problem is $O\left(t^{-1 / 2}\right)$ in the infinite domain and $O\left(t^{-1}\right)$ in the periodic case (see $[13,14]$ ). Therefore the use of an explicit scheme in a time-consistent approach may turn out to be extremely expensive.

To retain the characteristic of highly-resolved steady-state solutions by explicit second-order accurate TVD schemes without the disadvantage of slow convergence rate of explicit schemes, we considered in [11] the following two possibilities: (1) First obtain an approximation to the steady-state by using a conventional implicit scheme, and then use the second-order accurate TVD scheme as a "post-processor". (2) Use a first-order accurate implicit scheme in delta-formulation and replace the explicit operator by the explicit second-order accurate TVD scheme.

We have found (in one dimension) that both these strategies reduce the overall computational effort needed to obtain the steady-state solution of the 
explicit second-order accurate TVD scheme. Alternative (1) is a possible way of speeding up the convergence process by providing a better initial condition for the explicit second-order accurate TVD scheme. Alternative (2) can be viewed as a relaxation procedure to the steady-state solution. Numerical experiments of [11] show that the computational effort is not drastically decreased, although the stability limit is higher than the explicit counterpart.

Recently, Harten [3] has extended the class of explicit TVD schemes to a more general category which includes a one-parameter family of implicit second-order accurate schemes. Included in this class are the commonly used time-differencing schemes such as the backward Euler and the trapezoidal formula.

This paper is a sequel to [11]. In here we investigate the application to steady-state calculations of this newly developed implicit second-order accurate scheme that is unconditionally $T V D$. This scheme is guaranteed not to generate spurious oscillations for nonlinear scalar equations and constant coefficient systems. Numerical experiments show that this scheme has a rapid convergence rate, in addition to generating a highly-resolved approximation to the steady-state solution. We remark that all of the analysis on the new scheme is for the initial value problem. The numerical boundary conditions are not included.

In the present paper we stress applications rather than theory, and we refer the interested reader to [2,3] for more theoretical details. In the next section, we will briefly review the notion of $T V D$ schemes and describe the construction of the second-order accurate TVD scheme from a first-order accurate one for scalar one-dimensional hyperbolic conservation laws. The generalization to one-dimensional hyperbolic systems will be described in section III. A description of the algorithm and numerical results for the oneand two-dimensional compressible inviscid equations of gas dynamics will be presented in sections IV and V.

The paper is organized as follows:

I. Introduction

II. TVD Schemes for One-Dimensional Nonlinear Scalar Hyperbolic 
Conservation Laws

2.1 Explicit $T V D$ schemes

2.2 Implicit TVD Schemes

2.3 First-Order Accurate Backward Euler Implicit TVD Scheme

2.4 Conversion to Second-Order Accurate Schemes

2.5 Enhancement of Resolution by Artificial Compression

2.6 Linearized Version of the Implicit TVD Scheme

III. Generalization to One-Dimensional Hyperbolic Systems of Conservation Laws

IV. Applications to One-Dimensional Compressible Inviscid Equations of Gas Dynamics

4.1 Description of the Implicit Algorithm

4.2 Numerical Example

4.3 Discussion of Numerical Results

V. Applications to Two-Dimensional Compressible Inviscid Equations of Gas Dynamics

5.1 Numerical Fluxes in Two-Dimensions

5.2 Extension of the Explicit TVD Scheme by the Fractional Step Method

5.3 Extension of the Implicit TVD Scheme by the Alternating Direction Implicit $(A D I)$ Method

5.4 Numerical Example

5.5 Discussion of Numerical Results

VI. Concluding Remarks

\section{§2. TVD Schemes Por One-Dimensional Scalar Hyperbolic Conservation Laws}

Several techniques for the construction of nonlinear, explicit, second-order accurate, high-resolution, entropy satisfying schemes for hyperbolic conservation laws have been developed in recent years. See, for example, van Leer [7], Colella and Woodward [15], Harten [2], and Osher and Chakravarthy [9]. We can also view these schemes as shock-capturing algorithms based on either an exact or approximate Riemann solver. From the standpoint of numerical analysis, these schemes are TVD for nonlinear scalar hyperbolic conservation laws and for constant coefficient hyperbolic systems. Entropy satisfying TVD schemes have the property that they do not generate spurious 
oscillations and that the weak solutions are physical ones. The goal of constructing these highly nonlinear schemes is to simulate complex flow fields more accurately. TVD schemes are usually rather complicated to use compared with the conventional shock-capturing methods such as variants of the Lax-Wendroff scheme.

In [3]; Harten introduced the notation of implicit TVD schemes. To keep this paper somewhat self-contained, we will review the construction of the backward Euler TVD schemes for the initial value problem. This is the only unconditionally stable $T V D$ scheme belonging to the one-parameter family of $T V D$ schemes considered in [3]. Although the trapezoidal formula belongs to the same one-parameter family, the $C F L$ like requirement for this implicit scheme to be $T V D$ is 2 ; thus it is neither efficient nor practical for steadystate calculation. Before we proceed with the description of the construction, we will first give preliminaries on the definition of explicit and implicit TVD schemes and show a few examples.

\section{§2.1 Explicit TVD Schemes}

Consider the scalar hyperbolic conservation law

$$
\frac{\partial u}{\partial t}+\frac{\partial f(u)}{\partial x}=0
$$

where $a(u)=\partial f / \partial u$ is the characteristic speed. A general three-point explicit difference scheme in conservation form can be written as

$$
u_{j}^{n+1}=u_{j}^{n}-\lambda\left(\bar{f}_{j+1 / 2}^{n}-\vec{f}_{j-1 / 2}^{n}\right)
$$

where $\bar{f}_{j+1 / 2}^{n}=\bar{f}\left(u_{j}^{n}, u_{j+1}^{n}\right), \lambda=\Delta t / \Delta x$, with $\Delta t$ the time step, and $\Delta x$ the mesh size. Here, $u_{j}^{n}=u(j \Delta x, n \Delta t)$ and $\bar{f}$ is a numerical flux function. We require the numerical flux function $\bar{f}$ to be consistent with the conservation law in the following sense

$$
\bar{f}\left(u_{j}, u_{j}\right)=f\left(u_{j}\right)
$$


Consider a numerical scheme with numerical flux functions of the following form

$$
\bar{f}_{j+1 / 2}=\frac{1}{2}\left[f_{j}+f_{j+1}-Q\left(a_{j+1 / 2}\right) \Delta_{j+1 / 2} u\right]
$$

where $f_{j}=f\left(u_{j}\right), \Delta_{j+1 / 2} u=u_{j+1}-u_{j}$ and

$$
a_{j+1 / 2}= \begin{cases}\left(f_{j+1}-f_{j}\right) / \Delta_{j+1 / 2} u & \Delta_{j+1 / 2} u \neq 0 \\ a\left(u_{j}\right) & \Delta_{j+1 / 2} u=0\end{cases}
$$

Here $Q$ is a function of $a_{j+1 / 2}$ and $\lambda$. The function $Q$ is sometimes referred to as the coefficient of numerical viscosity. Figure (2.1) shows some examples for the possible choice of $Q$. Three familiar schemes with the numerical fluxes of the form (2.4) are:

(a) A form of the Lax-Wendroff (L-W) scheme with

$$
\bar{f}_{j+1 / 2}=\frac{1}{2}\left[f_{j}+f_{j+1}-\lambda\left(a_{j+1 / 2}\right)^{2} \Delta_{j+1 / 2} u\right]
$$

where $Q\left(a_{j+1 / 2}\right)=\lambda\left(a_{j+1 / 2}\right)^{2}$

(b) Lax-Friedrichs (L-F) scheme with

$$
\bar{f}_{j+1 / 2}=\frac{1}{2}\left[f_{j}+f_{j+1}-\Delta_{j+1 / 2} u\right]
$$

where $Q\left(a_{j+1 / 2}\right)=1$

(c) A generalization of the Courant-Isaacson-Rees (GCIR) scheme with

$$
\bar{f}_{j+1 / 2}=\frac{1}{2}\left[f_{j}+f_{j+1}-\left|a_{j+1 / 2}\right| \Delta_{j+1 / 2} u\right]
$$

where $Q\left(a_{j+1 / 2}\right)=\left|a_{j+1 / 2}\right|$

We define the total variation of a mesh function $u$ to be 


$$
T V(u)=\sum_{j=-\infty}^{\infty}\left|u_{j+1}-u_{j}\right|=\sum_{j=-\infty}^{\infty}\left|\Delta_{j+1 / 2} u\right|
$$

We say that the numerical scheme (2.2) is $T V D$ if

$$
T V\left(u^{n+1}\right) \leq T V\left(u^{n}\right)
$$

It can be shown that a sufficient condition for (2.2), together with (2.4), to be a $T V D$ scheme is [2]

$$
\begin{aligned}
& \lambda C_{j+1 / 2}^{-}=\frac{\lambda}{2}\left[-a_{j+1 / 2}+Q\left(a_{j+1 / 2}\right)\right] \geq 0 \\
& \lambda C_{j+1 / 2}^{+}=\frac{\lambda}{2}\left[a_{j+1 / 2}+Q\left(a_{j+1 / 2}\right)\right] \geq 0 \\
& \lambda\left(C_{j+1 / 2}^{-}+C_{j+1 / 2}^{+}\right)=\lambda Q\left(a_{j+1 / 2}\right) \leq 1
\end{aligned}
$$

Applying condition (2.10) or (2.11) to the above three examples, it can be easily shown that the L-W scheme is not a $T V D$ scheme, and the latter two schemes are TVD schemes. Note that there is a further distinction between the L-F scheme and GCIR scheme: the L-F scheme is consistent with an entropy inequality whereas the GCIR is not [6].

It should be emphasized that condition (2.11) is only a sufficient condition; i.e., schemes that fail this test might be still TVD. The L-W scheme, besides failing condition (2.11), does not satisfy (2.10).

\section{\$2.2 Implicit TVD Schemes}

Now we consider a one parameter family of three-point conservative schemes of the form

$$
u_{j}^{n+1}+\lambda \eta\left(\bar{f}_{j+1 / 2}^{n+1}-\bar{f}_{j-1 / 2}^{n+1}\right)=u_{j}^{n}-\lambda(1-\eta)\left(\bar{f}_{j+1 / 2}^{n}-\bar{f}_{j-1 / 2}^{n}\right)
$$


where $\eta$ is a parameter, $\lambda=\Delta t / \Delta x, \vec{f}_{j+1 / 2}^{n}=\bar{f}\left(u_{j}^{n}, u_{j+1}^{n}\right), \vec{f}_{j+1 / 2}^{n+1}=$ $\bar{f}\left(u_{j}^{n+1}, u_{j+1}^{n+1}\right)$, and $\bar{f}\left(u_{j}, u_{j+1}\right)$ is the numerical flux (2.4). This oneparameter family of schemes contain implicit as well as explicit schemes. When $\eta=0,(2.12)$ reduces to (2.2), the explicit method. When $\eta \neq 0$, (2.12) is an implicit scheme. For example: if $\eta=1 / 2$, the time differencing is the trapezoidal formula and if $\eta=1$, the time differencing is the backward Euler method. To simplify the notation, we will rewrite (2.12) as

$$
L \cdot u^{n+1}=R \cdot u^{n}
$$

where $L$ and $R$ are the following finite-difference operators:

$$
\begin{aligned}
(L \cdot u)_{j} & =u_{j}+\lambda \eta\left(\bar{f}_{j+1 / 2}-\bar{f}_{j-1 / 2}\right) \\
(R \cdot u)_{j} & =u_{j}-\lambda(1-\eta)\left(\bar{f}_{j+1 / 2}-\bar{f}_{j-1 / 2}\right)
\end{aligned}
$$

A sufficient condition for (2.12) to be a TVD scheme is that

$$
T V(R \cdot v) \leq T V(v)
$$

and

$$
T V(L \cdot v) \geq T V(v)
$$

A sufficient condition for (2.15) is the $C F L$-like condition

$$
\left|\lambda a_{j+1 / 2}\right| \leq \lambda Q\left(a_{j+1 / 2}\right) \leq \frac{1}{1-\eta}
$$

where $a_{j+1 / 2}$ is defined in equation (2.5). For a detailed proof of (2.15) and (2.16), see [3]. Observe that the backward Euler implicit scheme, $\eta=1$ in (2.12) is unconditionally $T V D$, while the trapezoidal formula, $\eta=1 / 2$ is TVD under the $C F L$-like restriction of 2 . The forward Euler explicit scheme, $\eta=0$ or (2.2), is $T V D$ under the $C F L$ condition of 1 . We remark that three-point conservative $T V D$ schemes of the form (2.12) are generally first-order accurate in space. When $\eta=1 / 2$, it is second-order accurate in time. 


\section{§2.3 First-Order Accurate Backward Euler Implicit TVD Scheme}

In this paper, we are only interested in efficient high resolution time dependent methods for steady-state calculations. The backward Euler implicit $T V D$ scheme is the best choice in this one-parameter family of TVD schemes. In this section we will review the proof that the backward Euler scheme is unconditionally $T V D$. In section 2.4 , we will decribe the technique of converting the first-order accurate unconditionally $T V D$ scheme (2.12) with $\eta=$ 1 into a second-order accurate one.

The backward Euler three-point scheme in conservative form can be written as

$$
u_{j}^{n+1}+\lambda\left(\bar{f}_{j+1 / 2}^{n+1}-\bar{f}_{j-1 / 2}^{n+1}\right)=u_{j}^{n}
$$

with

$$
\bar{f}\left(u_{j}, u_{j+1}\right)=\frac{1}{2}\left[f_{j}+f_{j+1}-Q\left(a_{j+1 / 2}\right) \Delta_{j+1 / 2} u\right]
$$

where

$$
a_{j+1 / 2}= \begin{cases}\left(f_{j+1}-f_{j}\right) / \Delta_{j+1 / 2} u & \Delta_{j+1 / 2} u \neq 0 \\ a_{j} & \Delta_{j+1 / 2} u=0 .\end{cases}
$$

For the purpose of this paper, the function $Q(z)$ in $(2.17 \mathrm{~b})$ is chosen to be

$$
Q(z)= \begin{cases}\frac{1}{2}\left(z^{2} / \delta+\delta\right) & |z|<\delta \\ |z| & |z| \geq \delta\end{cases}
$$

which is a nonvanishing, continuously differentiable approximation to $|z|$. This choice of $Q$ is the least dissipative among the class of three-point entropy satisfying $T V D$ schemes that we are considering (see figure (2.1)). Note that if $\delta=0$ in (2.17d), (i.e., $Q(z)=|z|)$ then (2.17a) is a first-order accurate, upstream differencing, backward-Euler scheme. In order to see the above (with a more familiar notation for upstream differencing representation) and to guide us for later development, we introduce the following notation

$$
C^{ \pm}(z)=\frac{1}{2}[Q(z) \pm z]
$$


and note that

$$
C^{ \pm}(z) \geq 0 \quad \text { for all } z
$$

Using (2.17c) and (2.18a), we can rewrite the numerical fluxes $\bar{f}_{j \pm 1 / 2}$ in $(2.17 \mathrm{~b})$ as

$$
\begin{aligned}
& \bar{f}_{j+1 / 2}=f_{j}-C^{-}\left(a_{j+1 / 2}\right) \Delta_{j+1 / 2} u \\
& \bar{f}_{j-1 / 2}=f_{j}-C^{+}\left(a_{j-1 / 2}\right) \Delta_{j-1 / 2} u
\end{aligned}
$$

It follows from (2.19) that (2.17) can be written in the form

$$
u_{j}^{n+1}-\lambda C^{-}\left(a_{j+1 / 2}^{n+1}\right) \Delta_{j+1 / 2} u^{n+1}+\lambda C^{+}\left(a_{j-1 / 2}^{n+1}\right) \Delta_{j-1 / 2} u^{n+1}=u_{j}^{n}
$$

Now, if $\delta=0$ in (2.17d), then $C \pm(z)=\frac{1}{2}(|z| \pm z)$, and (2.20) is a first-order accurate, upstream differencing, backward Euler implicit scheme. Equation (2.17) differs from the upstream spatial differencing (with $\delta=0$ ) by the addition of a so called numerical viscosity term with a coefficient $\delta>0 ; \delta$ has the dimensions of velocity. The addition of this viscosity term accomplishes two things: (i) it makes the numerical flux continuously differentiable, (ii) it rejects stationary expansion shocks (see [2] for more details). In general, a larger $\delta$ brings about improvement in entropy enforcement at the expense of some loss of resolution. However, from numerical experiments the dependence on $\delta$ is rather slight with $\delta=0.025,0.05$ and 0.125 .

We show now that $C \pm(z) \geq 0$ implies that the scheme (2.17) is unconditionally $T V D$ (i.e., condition (2.10) is satisfied), independent of the value of $\lambda=\Delta t / \Delta x$ in (2.17a).

To see that, we subtract (2.20) at $j$ from $(2.20)$ at $j+.1$ and get after rearranging terms that

$$
\begin{aligned}
& {\left[1+\lambda C_{j+1 / 2}^{-}+\lambda C_{j+1 / 2}^{+}\right] \Delta_{j+1 / 2} u^{n+1} } \\
= & \Delta_{j+1 / 2} u^{n}+\lambda C_{j-1 / 2}^{+} \Delta_{j-1 / 2} u^{n+1}+\lambda C_{j+3 / 2}^{-} \Delta_{j+3 / 2} u^{n+1}
\end{aligned}
$$


Here $C_{j+1 / 2}^{ \pm}=C^{ \pm}\left(a_{j+1 / 2}^{n+1}\right)$. Next we take the absolute value of (2.21a) and use (2.18b) and the triangle inequality to obtain

$$
\begin{aligned}
& {\left[1+\lambda C_{j+1 / 2}^{-}+\lambda C_{j+1 / 2}^{+}\right]\left|\Delta_{j+1 / 2} u^{n+1}\right| } \\
\leq & \left|\Delta_{j+1 / 2} u^{n}\right|+\lambda C_{j-1 / 2}^{+}\left|\Delta_{j-1 / 2} u^{n+1}\right|+\lambda C_{j+3 / 2}^{-}\left|\Delta_{j+3 / 2} u^{n+1}\right|
\end{aligned}
$$

Rearranging terms, we get

$$
\begin{aligned}
\left|\Delta_{j+1 / 2} u^{n+1}\right| & \leq\left|\Delta_{j+1 / 2} u^{n}\right| \\
& +\lambda\left[C_{j+3 / 2}^{-}\left|\Delta_{j+3 / 2} u^{n+1}\right|-C_{j+1 / 2}^{-}\left|\Delta_{j+1 / 2} u^{n+1}\right|\right] \\
& -\lambda\left[C_{j+1 / 2}^{+}\left|\Delta_{j+1 / 2} u^{n+1}\right|-C_{j-1 / 2}^{+}\left|\Delta_{j-1 / 2} u^{n+1}\right|\right]
\end{aligned}
$$

That is

$$
\left|\Delta_{j+1 / 2} u^{n+1}\right| \leq\left|\Delta_{j+1 / 2} u^{n}\right|+\lambda\left(\boldsymbol{\Xi}_{j+1}-\boldsymbol{\Xi}_{j}\right)
$$

where

$$
\mathrm{\Xi}_{j}=C_{j+1 / 2}^{-}\left|\Delta_{j+1 / 2} u^{n+1}\right|-C_{j-1 / 2}^{+}\left|\Delta_{j-1 / 2} u^{n+1}\right|
$$

Summing (2.21d) from $j=-\infty$ to $j=+\infty$, we obtain (2.10); thus proving that our backward Euler implicit scheme is unconditionally $T V D$.

We note that the TVD property does not depend on the particular form of the flux, but in general, is subject to a $C F L$ like restriction (2.16).

\section{\$2.4 Conversion to Second-Order Accurate Scheme}

Next, we want to briefly review the design principle behind the construction of second-order accurate TVD schemes. This is a rather general technique to convert a three-point first-order accurate (in space) TVD scheme (2.12) into 
a five-point second-order accurate (in both time and space, or just space) $T V D$ scheme of the same generic form. The design of high-resolution TVD schemes rests on the fact that the exact solution to (2.1) is TVD due to the phenomenon of propagation along characteristics, and is independent of the particular form of the flux $f(u)$ in (2.1). Similarly, the first-order accurate scheme is $T V D$ subject only to the $C F L$ like restriction (2.16), independent of the particular form of the flux. Thus to achieve second-order accuracy while retaining the $T V D$ property, we use the original TVD scheme with an appropriately modified flux $(f+g)$, i.e.,

$$
\begin{gathered}
u_{j}^{n+1}+\lambda\left(\tilde{f}_{j+1 / 2}^{n+1}-\tilde{f}_{j-1 / 2}^{n+1}\right)=u_{j}^{n} \\
\tilde{f}_{j+1 / 2}=\frac{1}{2}\left[f_{j}+f_{j+1}+g_{j}+g_{j+1}-Q\left(a_{j+1 / 2}+\gamma_{j+1 / 2}\right) \Delta_{j+1 / 2} u\right]
\end{gathered}
$$

where

$$
\gamma_{j+1 / 2}= \begin{cases}\left(g_{j+1}-g_{j}\right) / \Delta_{j+1 / 2} u & \Delta_{j+1 / 2} u \neq 0 \\ 0 & \Delta_{j+1 / 2} u=0\end{cases}
$$

The requirements on $g$ are: (i) The function $g$ should have a bounded $\gamma$ in (2.22c) so that (2.22a) is TVD with respect to the modified flux $(f+g)$. (ii) The modified scheme should be second-order accurate (except at points of extrema; i.e., $u_{j}=u_{j+1}$ ). In [2,3], Harten devised a recipe for $g$ that satisfies the above two requirements. We will use this particular form of $g$ for the discussion here. It can be written as

$$
\begin{aligned}
g_{j} & =S \cdot \max \left[0, \min \left(\sigma_{j+1 / 2}\left|\Delta_{j+1 / 2} u\right|, S \cdot \sigma_{j-1 / 2} \Delta_{j-1 / 2} u\right)\right] \\
S & =\operatorname{sign}\left(\Delta_{j+1 / 2} u\right)
\end{aligned}
$$

with $\sigma_{j+1 / 2}=\sigma\left(a_{j+1 / 2}\right)$ and we choose

$$
\sigma(z)=\frac{1}{2} Q(z) \geq 0
$$

for steady-state applications. It has the property that the steady-state solution is independent of $\Delta t$. Or, we choose 


$$
\sigma(z)=\frac{1}{2}\left(Q(z)+\lambda z^{2}\right) \geq 0
$$

for time accurate calculations. Note that if $\sigma(z)=\frac{1}{2}\left(Q(z)+\lambda z^{2}\right)$, then (2.22) is second-order accurate in both time and space; see [3]. For transient calculations, second-order accurate in time is preferred.

The form of $g$ in (2.22d) satisfies the following relations [3]:

$$
\begin{array}{r}
g_{j}=g\left(u_{j-1}, u_{j}, u_{j+1}\right), \quad g(u, u, u)=0 \\
\left|\gamma_{j+1 / 2}\right|=\left|g_{j+1}-g_{j}\right| /\left|u_{j+1}-u_{j}\right| \leq \sigma\left(a_{j+1 / 2}\right) \\
g=\Delta x \sigma(a) \frac{\partial u}{\partial x}+O\left((\Delta x)^{2}\right)
\end{array}
$$

Relation (2.23a) show s that the modified numerical flux (2.22b) is consistent with $f(u)$. Relation (2.23b) shows that the mean-value characteristic speed $\gamma_{j+1 / 2}(2.22 \mathrm{c})$ induced by the flux $g$ is uniformly bounded. Relation (2.23c) implies that (2.22b) is second-order accurate in space. The form of $g$ appears more complicated than it really is. The various test functions in $(2.22 \mathrm{~d})$ can be viewed as an automatic way of controlling the numerical flux function so that (2.22) is $T V D$.

The scheme (2.22) can be rewritten in the form (2.20) as

$$
u_{j}^{n+1}-\lambda C^{-}(a+\gamma)_{j+1 / 2}^{n+1} \Delta_{j+1 / 2} u^{n+1}+\lambda C^{+}(a+\gamma)_{j-1 / 2}^{n+1} \Delta_{j-1 / 2} u^{n+1}=u_{j}^{n}
$$

where $C^{ \pm}(a+\gamma)_{j \pm 1 / 2}^{n+1} \equiv C^{ \pm}\left(a_{j \pm 1 / 2}^{n+1}+\gamma_{j \pm 1 / 2}^{n+1}\right)$; i.e., $C^{ \pm}$is now a function of $(a+\gamma)$ instead of $a$. The modified scheme (2.22) is of the same generic form as the original first-order scheme (2.17). Therefore (2.22) is an upstream differencing scheme with respect to the characteristic field $(a+\gamma)$. Moreover, we have the following relation

$$
\operatorname{sign}(a+\gamma)=\operatorname{sign}(a)
$$

for $|z| \geq \delta$, with $z=a$ or $(a+\gamma)$. Hence (2.24) is also an upstream differencing scheme with respect to the original characteristic field $a(u)$. 
Because of (2.23a), the numerical flux (2.22b) of the second-order accurate $T V D$ scheme depends on four points; i.e., $\tilde{f}_{j+1 / 2}=\tilde{f}\left(u_{j-1}, u_{j}, u_{j+1}, u_{j+2}\right)$, and thus (2.22) is formally a five point scheme. We note, however, that

$$
\tilde{f}(v, u, u, w)=f(u)
$$

for all $v$ and $w$. Hence, for practical purposes, such as numerical boundary conditions, (2.22) can be regarded as essentially a three-point scheme.

We turn now to examine the behavior of TVD schemes around points of extrema, by considering their application to data where

$$
u_{j-1} \leq u_{j}=u_{j+1} \geq u_{j+2}
$$

In this case $g_{j}=g_{j+1}=0$ in (2.22d), and thus the numerical flux (2.22b) becomes identical to that of the original first-order accurate scheme (2.17b); consequently, the truncation error of (2.22) deteriorates to $O\left((\Delta x)^{2}\right)$ at $j$ and $j+1$. This behavior is common to all TVD schemes. We conclude that, for a second-order accurate scheme to be $T V D$, it has to have a mechanism that switches itself into a first-order accurate $T V D$ scheme at points of extrema. Because of the above property, second-order accurate TVD schemes are genuinely nonlinear, i.e., they are nonlinear even in the constant coefficient case.

Extension of the one-parameter family of three-point TVD schemes (2.12) to second-order TVD schemes follows the same procedure except (2.22f) becomes

$$
\sigma(z)=\frac{1}{2} Q(z)-\lambda(\eta-1 / 2) z^{2}
$$

\section{§2.5 Enhancement of Resolution by Artiflcial Compression}

Our technique to convert the first-order accurate $T V D$ scheme (2.12) into a second-order accurate one is closely related to the concept of artificial compression (see $[16,17]$ ).

Truncation error analysis shows that the first-order accurate scheme (2.12) is a second-order accurate approximation to solutions of the modified equation 


$$
u_{t}+f_{x}=\Delta x \frac{\partial}{\partial x}\left(\sigma(a) \frac{\partial u}{\partial x}\right)
$$

where $\sigma(a)$ is defined in $(2.22 \mathrm{e})$ or $(2.28)$

We note that the $C F L$ restriction (2.16) implies that $\sigma(a) \geq 0$; thus the right-hand side of (2.29) is a viscosity term. Hence the first-order accurate $T V D$ scheme (2.12) is a better approximation to the viscous equation (2.29) than it is to the original conservation law.

We obtain a second-order approximation to $\partial u / \partial t+\partial f / \partial x=0$ by applying the first-order scheme (2.12) to the modified flux $(f+g)$, where $g$ is an approximation to the right-hand side of (2.29); i.e.,

$$
g=\Delta x \frac{\partial}{\partial x}\left(\sigma(a) \frac{\partial u}{\partial x}\right)+O\left((\Delta x)^{2}\right)
$$

The application of the first-order scheme to $(f+g)$ has the effect of cancelling the error due to the numerical viscosity to $O\left((\Delta x)^{2}\right)$; thus $g$ is an "anti-diffusion" flux.

As to be expected, when we apply the first-order TVD scheme to $(f+$ $(1+\omega) g), \omega>0$, rather than to $(f+g)$, we find that the resolution of discontinuities improves with increasing $\omega$. This observation allows us to use the notion of artificial compression to enhance the resolution of discontinuities computed by the second-order accurate TVD scheme (2.22). This is done by increasing the size of $g$ in $(2.22 \mathrm{~d})$ by adding a term that is $O\left((\Delta x)^{2}\right)$ in regions of smoothness, e.g.,

$$
\tilde{g}_{j}=\left(1+\omega \theta_{j}\right) g_{j}, \quad \omega>0
$$

with

$$
\theta_{j}=\frac{\left|\Delta_{j+1 / 2} u-\Delta_{j-1 / 2} u\right|}{\left|\Delta_{j+1 / 2} u\right|+\left|\Delta_{j-1 / 2} u\right|}
$$

Using $\tilde{g}_{j}(2.31)$ instead of $g_{j}$ makes the numerical characteristic speed more convergent, and therefore improves the resolution of computed shocks. Since $\theta=O(\Delta x)$, this change does not adversely affect the order of accuracy of 
the scheme. See [2] for more details. From numerical experiments, $\omega=2$ seems is a good choice.

We remark that applying too much artificial compression in a region of expansion (i.e., divergence of the characteristic field $a=\delta f / \partial u$ ) may result in violation of the entropy condition. Hence when applying artificial compression, one has to either turn it off in regions of expansion, or to limit the size of $\omega$ in (2.31a), say, by the value that makes (2.22) with (2.31a) third-order accurate (in regions of monotonicity).

\section{\$2.6 Linearized Version of the Implicit TVD Scheme}

In order to solve for $u^{n+1}$ for this first- or second-order implicit scheme, we have to solve a set of nonlinear algebraic equations. The iterations needed to solve this system of nonlinear equations, say by Newton's method at each time-step, may not compensate for the savings gained by the ability to take larger time-steps.

To overcome this obstacle, we will present two ways of linearizing the implicit TVD scheme. The first method will preseve the conservative form of the differencing scheme but the resulting scheme may no longer be TVD or unconditionally $T V D$. The second method will destroy the conservative property but preserve its unconditionally $T V D$ property. We will refer to the first method as the linearized conservative implicit (LCI) form, and the latter the linearized nonconservative implicit (LNI) form. The LNI is mainly useful for steady-state calculations, since the scheme is only conservative after the solution reaches steady-state. On the other hand, we have the advantage of stability of an unlimited $C F L$ number. Note that the procedure of obtaining the LCI and LNI forms are applicable to both the firstand secondorder accurate Implicit TVD schemes. We will discuss the LCI and LNI for the second-order accurate one. To get the LCI and LNI for the first-order accurate $T V D$ scheme, one simply sets $q=\gamma=0$ in the second-order form. Rewrite equation (2.22b) as

$$
\begin{aligned}
\tilde{f}_{j+1 / 2}^{n+1} & =\frac{1}{2}\left[f_{j}^{n+1}+f_{j+1}^{n+1}\right] \\
& +\frac{1}{2}\left[\left(\frac{g_{j}+g_{j+1}}{\Delta_{j+1 / 2} u}\right) \Delta_{j+1 / 2} u-Q\left(a_{j+1 / 2}+\gamma_{j+1 / 2}\right) \Delta_{j+1 / 2} u\right]^{n+1}
\end{aligned}
$$


and use a local Taylor expansion about $u^{n}$

$$
f^{n+1}-f^{n}=a^{n}\left(u^{n+1}-u^{n}\right)+O\left(\Delta t^{2}\right)
$$

where $a=\partial f / \partial u$. Applying the first-order approximation of (2.33) and locally linearizing the coefficients of $\Delta_{j+1 / 2} u$ in the second and third terms on the right-hand-side by dropping the time index from $(n+1)$ to $n$, we get the LCI form

$$
\begin{aligned}
& u_{j}^{n+1}-\frac{\lambda}{2}\left\{\bar{G}+\left(\beta_{j+1 / 2}^{n}-Q\left(a_{j+1 / 2}^{n}+\gamma_{j+1 / 2}^{n}\right)\right) \Delta_{j+1 / 2} u^{n+1}\right. \\
& \left.-\left(\beta_{j-1 / 2}^{n}-Q\left(a_{j-1 / 2}^{n}+\gamma_{j-1 / 2}^{n}\right)\right) \Delta_{j-1 / 2} u^{n+1}\right\}=u_{j}^{n}
\end{aligned}
$$

where

$$
\begin{aligned}
\bar{G} & =a_{j+1}^{n} u_{j+1}^{n}-a_{j-1}^{n} u_{j-1}^{n} \\
\beta_{j+1 / 2}^{n} & =\left(\frac{g_{j}+g_{j+1}}{\Delta_{j+1 / 2} u}\right)^{n}
\end{aligned}
$$

Denote $d_{j}=u_{j}^{n+1}-u_{j}^{n}$, i.e., the delta notation, (2.34) can be rewritten

$$
E_{1} d_{j-1}+E_{2} d_{j}+E_{3} d_{j+1}=-\lambda\left[\tilde{f}_{j+1 / 2}^{n}-\tilde{f}_{j-1 / 2}^{n}\right]
$$

where

$$
\begin{aligned}
\bar{E}_{1}= & \frac{\lambda}{2}\left[-a_{j-1}^{n}+\beta_{j-1 / 2}^{n}-Q\left((a+\gamma)_{j-1 / 2}^{n}\right)\right] \\
\bar{E}_{2}=1 & +\frac{\lambda}{2}\left[-\beta_{j+1 / 2}^{n}+Q\left((a+\gamma)_{j+1 / 2}^{n}\right)-\beta_{j-1 / 2}^{n}\right. \\
& \left.+Q\left((a+\gamma)_{j-1 / 2}^{n}\right)\right] \\
\bar{E}_{3}=\frac{\lambda}{2} & {\left[a_{j+1}^{n}+\beta_{j+1 / 2}^{n}-Q\left((a+\gamma)_{j+1 / 2}^{n}\right)\right] }
\end{aligned}
$$


and $\tilde{f}_{j+1 / 2}^{n}$ is $(2.22 \mathrm{~b})-(2.22 \mathrm{e})$ calculated at the time level $n$. At this writing, we do not have any analysis to show that the LCI form is still TVD.

The LNI form is obtained simply by replacing the coefficients $(C \pm)^{n+1}$ with $(C \pm)^{n}$ in (2.24), i.e.,

$u_{j}^{n+1}-\lambda C^{-}(a+\gamma)_{j+1 / 2}^{n} \Delta_{j+1 / 2} u^{n+1}+\lambda C^{+}(a+\gamma)_{j-1 / 2}^{n} \Delta_{j-1 / 2} u^{n+1}=u_{j}^{n}$

Since $C \pm \geq 0$, it follows from (2.21) that (2.36) is unconditionally $T V D$.

In delta form notation, (2.36) can be rewritten as

$$
\begin{aligned}
{\left[1-\lambda C_{j+1 / 2}^{-} \Delta_{j+1 / 2}\right.} & \left.+\lambda C_{j-1 / 2}^{+} \Delta_{j-1 / 2}\right]\left(u^{n+1}-u^{n}\right) \\
& =-\lambda\left[\tilde{f}_{j+1 / 2}^{n}-\tilde{f}_{j-1 / 2}^{n}\right]
\end{aligned}
$$

where the left-hand-side equals

$$
d_{j}-\lambda C_{j+1 / 2}^{-} \Delta_{j+1 / 2} d+\lambda C_{j-1 / 2}^{+} \Delta_{j-1 / 2} d
$$

with $d_{j}=u_{j}^{n+1}-u_{j}^{n}, \Delta_{j+1 / 2} d=d_{j+1}-d_{j}$, and $C_{j+1 / 2}^{ \pm} \equiv$ $C \pm(a+\gamma)_{j+1 / 2}^{n} \equiv C \pm\left(a_{j+1 / 2}^{n}+\gamma_{j+1 / 2}^{n}\right)$. Rearranging terms, we get

$$
\bar{E}_{1} d_{j-1}+\bar{E}_{2} d_{j}+\bar{E}_{3} d_{j+1}=-\lambda\left[\tilde{f}_{j+1 / 2}^{n}-\tilde{f}_{j-1 / 2}^{n}\right]
$$

with

$$
\begin{aligned}
& \bar{E}_{1}=-\lambda C^{+}(a+\gamma)_{j-1 / 2}^{n} \\
& \bar{E}_{2}=1+\lambda\left[C^{-}(a+\gamma)_{j+1 / 2}^{n}+C^{+}(a+\gamma)_{j-1 / 2}^{n}\right] \\
& \bar{E}_{3}=-\lambda C^{-}(a+\gamma)_{j+1 / 2}^{n}
\end{aligned}
$$


Again, $\tilde{f}_{j+1 / 2}^{n}$ is $(2.22 \mathrm{~b})-(2.22 \mathrm{e})$ calculated at the time-level $n$. It follows from $(2.22 b)$ and (2.37a) that the steady-state solution of (2.37) is

(i) consistent with the conservation form,

(ii) a spatially second-order accurate approximation to the steady-state of the partial differential equation,

(iii) independent of the time-step $\Delta t$ used in the iterations.

Moreover, the iteration matrix associated with (2.38) is a diagonally dominant, tridiagonal matrix. Note that this linearized construction is not trivial, since the second-order method is a five-point scheme. Normally the matrix associated with (2.38) could have been a block pentadiagonal matrix. As mentioned before, (2.36) or (2.38) is not in conservation form and therefore should not be used to approximate time-dependent solutions (transient solutions). However, it is a suitable scheme for the calculation of steady-state solutions. The rest of the paper will be devoted only to the LNI form.

\section{§3. Generalization to One-Dimensional Hyperbolic System of Conservation Laws}

At the present development, the concept of $T V D$ schemes, like monotone schemes is only defined for nonlinear scalar conservation laws or constant coefficient hyperbolic systems. The main difficulty stems from the fact that, unlike the scalar case, the total variation in $x$ of the solution to the system of nonlinear conservation laws is not necessarily a monotonic decreasing function of time. The solution may actually increase at moments of interaction between waves. Not knowing a diminishing functional that bounds the totalvariation in $\mathrm{x}$ in the system case, makes it impossible to fully extend the theory of the scalar case to the system case. What we can do at the moment is to extend the new scalar TVD scheme to system cases so that the resulting scheme is $T V D$ for the "locally frozen" constant coefficient system. To accomplish this, we define at each point a "local" system of characteristic fields. This extension technique is a somewhat generalized version of the procedure suggested by Roe in [18].

Now, we briefly describe the above approach of extending the second-order accurate $T V D$ schemes to hyperbolic systems of conservation laws 


$$
\frac{\partial U}{\partial t}+\frac{\partial F(U)}{\partial x}=0, \quad A(U)=\frac{\partial F}{\partial U}
$$

Here $U$ and $F(U)$ are column vectors of $m$ components and $A(U)$ is the Jacobian matrix. The assumption that (3.1) is hyperbolic implies that $A(U)$ has real eigenvalues $a^{l}(U)$ and a complete set of right-eigenvectors $R^{l}(U)$, $l=1, \ldots, m$. Hence the matrix

$$
R(U)=\left(R^{1}(U), . ., R^{m}(U)\right)
$$

is invertible. The rows $L^{1}(U), \ldots, L^{m}(U)$ of $R(U)^{-1}$, constitute an orthornormal set of left eigenvectors of $A(U)$; thus

$$
R^{-1} A R=\operatorname{diag}\left(a^{l}\right)
$$

Here $\operatorname{diag}\left(a^{l}\right)$ denotes a diagonal matrix with diagonal elements $a^{l}$.

We define characteristic variables $W$ with respect to the state $U$ by

$$
W=R^{-1} U
$$

In the constant coefficient case (3.1) decouples into $m$ scalar equations for the characteristic variables

$$
\frac{\partial w^{l}}{\partial t}+a^{l} \frac{\partial w^{l}}{\partial x}=0, \quad a^{l}=\text { constant }
$$

This offers a natural way of extending a scalar scheme to a constant coefficient system by applying it "scalarly" to each of the $m$ scalar characteristic equations (3.4).

Let $U_{j+1 / 2}$ denote some symmetric average of $U_{j}$ and $U_{j+1}$ (to be discussed later); i.e.,

$$
U_{j+1 / 2}=\Psi\left(U_{j}, U_{j+1}\right)
$$

Let $a_{j+1 / 2}^{l}, R_{j+1 / 2}^{l}, L_{j+1 / 2}^{l}$ denote the respective quantities of $a^{l}, R^{l}, L^{l}$ related to $A\left(U_{j+1 / 2}\right)$. Let $w^{l}$ be the vector elements of $W$, and let $\alpha_{j+1 / 2}^{l}=$ $w_{j+1}^{l}-w_{j}^{l}$ be the component of $\Delta_{j+1 / 2} U=U_{j+1}-U_{j}$ in the lth characteristic direction; i.e., 


$$
\Delta_{j+1 / 2} U=R_{j+1 / 2} \alpha_{j+1 / 2} ; \quad \alpha_{j+1 / 2}=R_{j+1 / 2}^{-1} \Delta_{j+1 / 2} U
$$

With the above notations, we can apply scheme (2.22) scalarly to each of the locally defined (frozen coefficient) characteristic variables of (3.1) as follows:

$$
\begin{gathered}
U_{j}^{n+1}+\lambda\left(\tilde{F}_{j+1 / 2}^{n+1}-\tilde{F}_{j-1 / 2}^{n+1}\right)=U_{j}^{n}, \\
\tilde{F}_{j+1 / 2}=\frac{1}{2}\left(F_{j}+F_{j+1}\right) \\
+\frac{1}{2} \sum_{l=1}^{m}\left[g_{j}^{l}+g_{j+1}^{l}-Q\left(a_{j+1 / 2}^{l}+\gamma_{j+1 / 2}^{l}\right) \alpha_{j+1 / 2}^{l}\right] R_{j+1 / 2}^{l}
\end{gathered}
$$

where

$$
\begin{aligned}
g_{j}^{l} & =S \cdot \max \left[0, \min \left(\sigma_{j+1 / 2}^{l}\left|\alpha_{j+1 / 2}^{l}\right|, S \cdot \sigma_{j-1 / 2}^{l} \alpha_{j-1 / 2}^{l}\right)\right] \\
S & =\operatorname{sign}\left(\alpha_{j+1 / 2}^{l}\right)
\end{aligned}
$$

and

$$
\gamma_{j+1 / 2}^{l}= \begin{cases}\left(g_{j+1}^{l}-g_{j}^{l}\right) / \alpha_{j+1 / 2}^{l} & \alpha_{j+1 / 2}^{l} \neq 0 \\ 0 & \alpha_{j+1 / 2}^{l}=0\end{cases}
$$

Here $\sigma_{j+1 / 2}^{l}=\sigma\left(a_{j+1 / 2}^{l}\right)$ where $\sigma(z)$ is (2.22e) and $\alpha_{j+1 / 2}^{l}$ is (3.6). The corresponding $\tilde{g}_{j}$ in (2.31) for the added artificial compression term is

$$
\tilde{g}_{j}^{l}=\left(1+\omega^{l} \theta_{j}^{l}\right) g_{j}^{l}, \quad \omega^{l}>0
$$

with

$$
\theta_{j}^{l}=\frac{\left|\Delta_{j+1 / 2} u-\Delta_{j-1 / 2} u\right|}{\left|\Delta_{j+1 / 2} u\right|+\left|\Delta_{j-1 / 2} u\right|}
$$


The $\omega^{l}$ can be different from one characteristic field to another.

Similarly, we generalize the linearized nonconservative implicit (LNI) form (2.37) to the system case by

$$
\begin{aligned}
{\left[I-\lambda J \overline{j+1 / 2}_{j+1 / 2}\right.} & \left.+\lambda J_{j-1 / 2}^{+} \Delta_{j-1 / 2}\right]\left(U^{n+1}-U^{n}\right) \\
& =-\lambda\left[\tilde{F}_{j+1 / 2}^{n}-\tilde{F}_{j-1 / 2}^{n}\right]
\end{aligned}
$$

or

$$
\tilde{E}_{1} D_{j-1}+\tilde{E}_{2} D_{j}+\tilde{E}_{3} D_{j+1}=-\lambda\left[\tilde{F}_{j+1 / 2}^{n}-\tilde{F}_{j-1 / 2}^{n}\right]
$$

with

$$
\begin{aligned}
& \tilde{E}_{1}=-\lambda J_{j-1 / 2}^{+} \\
& \tilde{E}_{2}=I+\lambda\left[J_{j+1 / 2}^{-}+J_{j-1 / 2}^{+}\right] \\
& \tilde{E}_{3}=-\lambda J_{j+1 / 2}^{-}
\end{aligned}
$$

and

$$
\begin{gathered}
J_{j+1 / 2}^{ \pm}=R_{j+1 / 2}^{n} \operatorname{diag}\left(C^{ \pm}\left(a^{l}+\gamma^{l}\right)_{j+1 / 2}^{n}\right)\left(R^{-1}\right)_{j+1 / 2}^{n} \\
D_{j}=U_{j}^{n+1}-U_{j}^{n}
\end{gathered}
$$

where the left hand side of (3.8a) is equal to

$$
D_{j}-\lambda J_{j+1 / 2}^{-} \Delta_{j+1 / 2} D+\lambda J_{j-1 / 2}^{+{ }_{j-1 / 2}} \Delta_{j-1}
$$

with $\Delta_{j+1 / 2} D=D_{j+1}-D_{j}$.

In the constant coefficient case where $A(U)=$ constant, both (3.7) and (3.8) are TVD by construction. However, they are not identical; equation (3.7) is fully nonlinear while (3.8) is a version with a linearized left-hand-side.

Note that the total variation for the vector mesh function $U$ of the constant coefficient_case is defined as 


$$
T V(U)=\sum_{j=-\infty}^{\infty} \sum_{l=1}^{m}\left|\alpha_{j+1 / 2}^{l}\right|
$$

The particular form of averaging in (3.5) is essential if we require the scheme (3.7) for $m=1$ to be identical to the scalar scheme of section 2, since we have to choose (3.5) so that $a_{j+1 / 2}^{l}$ is the same as the mean value in equation $(2.17 \mathrm{c})$. This can be accomplished by taking the eigenvalues $a_{j+1 / 2}^{l}$ and the eigenvectors $R_{j+1 / 2}^{l}$ in (3.2) to be those of $A\left(U_{j}, U_{j+1}\right)$, where $A\left(U_{j}, U_{j+1}\right)$ is the mean value Jacobian. This matrix should satisfy

(i) $\quad F(U)-F(V)=A(U, V)(U-V)$

(ii) $A(U, U)=A(U)$

(iii) $A(U, V)$ has real eigenvalues and a complete set of eigenvectors.

Roe in [18] constructs a mean value Jacobian for the Euler equations of gas dynamics of the form $A(U, V)=A(\Psi(U, V))$, where $\Psi(U, V)$ is some particular average. We will discuss Roe's mean value Jacobian in the next two sections.

\section{§4. Applications to One-Dimensional Compressible Inviscid Equations of Gas Dynamics}

In this section we describe how to apply the implicit TVD scheme (3.8) to the compressible inviscid equations of gas dynamics (Euler equations). Included in this section are: (i) a detailed description of each of the terms of equations (3.5) to (3.8) for the Euler equations, (ii) Roe's special form for $A\left(U_{j}, U_{j+1}\right)$, (iii) an algorithm to compute $U_{j}^{n+1}$, (iv) a description of the numerical example, and (v) a discussion of the numerical results.

\section{§4.1 Description of Algorithm}

In one spatial dimension, the Euler equations of gas dynamics can be written in the conservative form as 


$$
\frac{\partial U}{\partial t}+\frac{\partial F(U)}{\partial x}=0
$$

where

$$
U=\left[\begin{array}{c}
\rho \\
m \\
e
\end{array}\right], \quad F=\left[\begin{array}{c}
m \\
m^{2} / \rho+p \\
(e+p) m / \rho
\end{array}\right]
$$

Here $U$ is the vector of conservative variables, $F$ is the flux vector, and $m=$ $\rho u$. The primitive variables are the density $\rho$, the velocity $u$, and the pressure $p$. The total energy per unit volume $e$, is defined as

$$
e=\rho \epsilon+\rho u^{2} / 2
$$

with $\epsilon$ as the internal energy per unit mass. The pressure $p$ for a perfect gas is defined as

$$
p=(\gamma-1)\left[e-m^{2} / 2 p\right]
$$

where $\gamma$ is the ratio of specific heats and should not be confused with the $\gamma_{j+1 / 2}$ in $(2.22 \mathrm{c})$ or $\gamma_{j+1 / 2}^{l}$ in (3.7d).

Let $A$ denote the Jacobian matrix $\partial F(U) / \partial U$ whose eigenvalues are

$$
\left(a^{1}, a^{2}, a^{3}\right)=(u-c, u, u+c)
$$

where $c$ is the local speed of sound. The right eigenvectors of $A$ form the matrix $R=\left(R^{1}, R^{2}, R^{3}\right)$ given by

$$
\begin{gathered}
R=\left[\begin{array}{ccc}
1 & 1 & 1 \\
u-c & u & u+c \\
H-u c & \frac{1}{2} u^{2} & H+u c
\end{array}\right] \\
H=\frac{c^{2}}{\gamma-1}+\frac{u^{2}}{2}
\end{gathered}
$$

and

$$
R^{-1}=\left[\begin{array}{ccc}
\frac{1}{2}\left(b_{1}+u / c\right) & \frac{1}{2}\left(-b_{2} u-1 / c\right) & \frac{1}{2} b_{2} \\
1-b_{1} & b_{2} u & -b_{2} \\
\frac{1}{2}\left(b_{1}-u / c\right) & \frac{1}{2}\left(-b_{2} u+1 / c\right) & \frac{1}{2} b_{2}
\end{array}\right]
$$


with

$$
\begin{aligned}
& b_{1}=b_{2} \frac{u^{2}}{2} \\
& b_{2}=\frac{\gamma-1}{c^{2}}
\end{aligned}
$$

Let the grid spacing be denoted by $\Delta x$ such that $x=j \Delta x$. Using the same notation as in section 3 , the vector $\alpha$ of equation (3.6) is

$$
\left[\begin{array}{c}
\alpha_{j+1 / 2}^{1} \\
\alpha_{j+1 / 2}^{2} \\
\alpha_{j+1 / 2}^{3}
\end{array}\right]=\left[\begin{array}{c}
(a a-b b) / 2 \\
\Delta_{j+1 / 2} \rho-a a \\
(a a+b b) / 2
\end{array}\right]
$$

where

$$
\begin{aligned}
a a & =\frac{\gamma-1}{c_{j+1 / 2}^{2}}\left[\Delta_{j+1 / 2} e+\frac{u_{j+1 / 2}^{2}}{2} \Delta_{j+1 / 2} \rho-u_{j+1 / 2} \Delta_{j+1 / 2} m\right] \\
b b & =\left[\Delta_{j+1 / 2} m-u_{j+1 / 2} \Delta_{j+1 / 2} \rho\right] / c_{j+1 / 2}
\end{aligned}
$$

The simplest form of $U_{j+1 / 2}$ is

$$
U_{j+1 / 2}=\left(U_{j+1}+U_{j}\right) / 2
$$

Roe in [18] uses a special form of averaging that has the computational advantage of perfectly resolving stationary discontinuities. Roe's averaging takes the following form

$$
\begin{aligned}
u_{j+1 / 2} & =\frac{\bar{D} u_{j+1}+u_{j}}{\bar{D}+1} \\
H_{j+1 / 2} & =\frac{\bar{D} H_{j+1}+H_{j}}{\bar{D}+1} \\
c_{j+1 / 2}^{2} & =(\gamma-1)\left(H_{j+1 / 2}-\frac{1}{2} u_{j+1 / 2}^{2}\right) \\
\bar{D} & =\sqrt{\rho_{j+1} / \rho_{j}} \\
H & =\frac{\gamma p}{(\gamma-1) \rho}+\frac{1}{2} u^{2}
\end{aligned}
$$


To use Roe's averaging, we compute $u_{j+1 / 2}, c_{j+1 / 2}$ in (4.2)-(4.4) by (4.6). In the numerical experiments for the one-dimensional test problem, we use Roe's averaging.

Given $U_{j}^{n}$, for all $j$, we now list the operations needed to calculate $U_{j}^{n+1}$ (assuming a fixed $C F L$ number as input):

(i) compute $u_{j}=m_{j} / \rho_{j}$ and $p_{j}$

(ii) compute $u_{j+1 / 2}$ and $c_{j+1 / 2}$ from (4.5) or (4.6), calculate

$$
M=\max _{j}\left(\left|u_{j+1 / 2}\right|+c_{j+1 / 2}\right)
$$

evaluate $\alpha_{j+1 / 2}^{l}, l=1,2,3$ by (4.4a), and define

$$
\lambda=\Delta t / \Delta x=\mu / M
$$

where $\mu$ is the prescribed $C F L$ number as input

(iii) compute $a_{j+1 / 2}^{l}$ by (4.2); $g_{j+1 / 2}^{l}$ by (3.7c)

(iv) compute $\gamma_{j+1 / 2}^{l}$ by (3.7d), and $\tilde{F}_{j+1 / 2}$ by (3.7b) and relation (4.3a)

(v) compute $C^{ \pm}\left(a_{j+1 / 2}^{l}+\gamma_{j+1 / 2}^{l}\right)$ by (2.18a) (with $\left.z=a_{j+1 / 2}^{l}+\gamma_{j+1 / 2}^{l}\right)$

(vi) compute $J_{j+1 / 2}^{+}$by (3.8f), and $\tilde{E}_{1}, \tilde{E}_{2}$ and $\tilde{E}_{3}$, by (3.8c)-(3.8e)

(vii) solve the tridigonal system (3.8b) for $D_{j}$ and then compute $U_{j}^{n+1}$ from $(3.8 \mathrm{~g})$.

\section{$\S 4.2$ Numerical Example}

For the numerical experiments, a quasi-one-dimensional nozzle problem was selected. The governing equations for the nozzle problem can be written as

$$
\frac{\partial \bar{U}}{\partial t}+\frac{\partial F(\bar{U})}{\partial x}+H(\bar{U})=0
$$

where 


$$
\bar{U}=\left[\begin{array}{c}
\rho \kappa \\
m \kappa \\
e \kappa
\end{array}\right], \quad F=\left[\begin{array}{c}
m \kappa \\
\left(m^{2} / \rho+p\right) \kappa \\
(e+p) m \kappa / \rho
\end{array}\right], \quad H(\bar{U})=\left[\begin{array}{c}
0 \\
p \frac{\partial \kappa}{\partial x} \\
0
\end{array}\right]
$$

with $\kappa$, the area of the nozzle a function of $x$. The nozzle we consider is a divergent nozzle [19] (figure 4.1) with

$$
\kappa(x)=1.398+0.347 \tanh (0.8 x-4)
$$

The steady flow condition were supersonic inflow, subsonic outflow with a shock. In all of the calculations the computational domain was $0 \leq x \leq 10$. We used a very coarse mesh spacing of $\Delta x=0.5$ (i.e., 20 spatial intervals), to evaluate the resolution of the scheme.

\section{Initial Conditions:}

We use linear interpolation between the exact steady-state boundary values as initial conditions.

\section{Analytical Boundary Conditions:}

We specified all three conservative variables $\rho, u$ and $e$ for the supersonic inflow, and the variable $e$ for the subsonic outflow.

\section{Numerical Boundary Conditions:}

We used zeroth- or first-order space extrapolation to obtain the numerical boundary conditions for the unknown flow variables $(\rho$ and $m)$ at the outflow boundary. Since the spatially second-order accurate TVD scheme is a fivepoint scheme, we also need the values of $g_{j}$ and $\theta_{j}$ on both boundaries. For convenience, we will use zeroth-order space extrapolation for these values.

\section{\$4.3 Discussion of Numerical Results}

All of the computations for the quasi-one-dimensional nozzle problem were done in single precision on the VAX 11/780 computer (a 6 digit machine). To illustrate the stability and/or accuracy of the LNI form of the implicit $T V D$ scheme (3.8b), we compare in figure (4.2) the computed results with the explicit TVD scheme (forward Euler in time, obtained by setting $\tilde{E}_{1}=0$, $\tilde{E}_{2}=I$, and $\tilde{E}_{3}=0$ in equation (3.8b)), the first-order flux-vector splitting scheme [20], and a conventional implicit method using backward Euler in time and central spatial differencing with an added fourth-order explicit, numerical 
dissipation [21]. Figure (4.2a) shows the numerical result of the explicit TVD scheme. It took approximately 700 steps to converge to the steady-state with a fixed $C F L=0.8$. Figure (4.2b) shows the computation of the LNI form of the second-order accurate implicit TVD scheme with $C F L=10^{6}$ after 25 steps. Both the explicit and implicit TVD schemes produce similar high resolution solutions (with $\delta=0.125$ in equation (2.17d)). From numerical experiments, we found that artificial compression is not necessary for the one-dimensional problem.

The convergence criterion is based on where all the terms on the right-hand side of equation (3.8b) are less than or equal to $10^{-4}$. The implicit $T V D$ method requires approximately triple the CPU time per time step more than the explicit $T V D$ method but results in enhanced convergence rate.

We have only used the nonconservative linearized delta form of the implicit $T V D$ method for numerical experiments. A steady-state solution can be reached in 25-30 steps with $C F L$ ranges from $10^{6}-10^{7}$. The steady-state solution profiles are independent of the $C F L$ number. The number of steps for convergence monotonically decreases as the $C F L$ number increases. However, the reduction in the number of steps is less pronounced for $C F L$ number in the range from $10^{3}$ to $10^{6}$. There are four primary factors affecting the convergence rate for $C F L$ numbers higher than $10^{3}$ : (1) the initial condition ( or the initial guess), (2) the numerical boundary conditions, (3) the interaction of nonlinear waves and (4) the machine accuracy of the VAX $11 / 780$. The influence of the above four factors will be the subject of a future investigation.

Figure (4.2c) shows the converged solution by a conventional implicit method with $C F L=10$. The oscillation near the shock is typical of a three-point central spatial difference scheme. The experimentally determined maximum $C F L$ number is around 10 with the above initial and numerical boundary conditions. (We can improve the stability by adding an implicit second-order numerical dissipation term.) Figure (4.2d) shows the converged solution by the first-order flux-vector splitting method [20] with $C F L=10^{6}$. Again, the steady-state is reached after $25-30$ time steps. The solution is very smeared but is independent of the $C F L$ number. From the results, we can see a definite improvement in shock resolution by the TVD schemes over the conventional methods. The implicit TVD scheme requires approximately $80 \%$ more CPU time per time step than the conventional implicit method. Moreover, there is a dramatic increase in convergence rate of the implicit $T V D$ scheme over the explicit TVD scheme. 


\section{§5. Applications to Two-Dimensional Compressible Inviscid Equations of Gas Dynamics}

In this section we describe how to formally extend the one-dimensional implicit TVD scheme to an Alternating Direction Implicit $(A D I)$ version for the two-dimensional compressible inviscid equations of gas dynamics (Euler equations). This is a formal extension of the TVD scheme from one dimension to two dimensions. At the present state of development, there is not yet a similar theoretical analysis of the TVD properties for two-dimensional hyperbolic equations. Included in this section are: (i) a detailed description of the analogue of each of the terms of equations (4.2) to (4.6) for the two-dimensional Euler equations, (ii) a discussion on the extension of the explicit and implicit TVD schemes to two-dimensions, (iii) Roe's form for the Jacobian matrices $A\left(U_{j, k}, U_{j+1, k}\right)$ and $B\left(U_{j, k}, U_{j+1, k}\right)$, (iv) an algorithm to compute $U_{j, k}^{n+1}$ by the $A D I$ approach, (v) a description of a numerical example, and (vi) a discussion of the numerical results.

\section{\$5.1 Numerical Fluxes in Two-Dimensions}

In two spatial dimensions, the Euler equations of gas dynamics can be written in the conservative form as

$$
\frac{\partial U}{\partial t}+\frac{\partial F(U)}{\partial x}+\frac{\partial G(U)}{\partial y}=0
$$

where

$$
U=\left[\begin{array}{c}
\rho \\
m \\
n \\
e
\end{array}\right], \quad F=\left[\begin{array}{c}
m \\
m^{2} / \rho+p \\
m v \\
(e+p) m / \rho
\end{array}\right], \quad G=\left[\begin{array}{c}
n \\
n u \\
n^{2} / \rho+p \\
(e+p) n / \rho
\end{array}\right]
$$

with $m=\rho u$ and $n=\rho v$. The primitive variables are the density $\rho$, the velocity components $u$ and $v$, and the pressure $p$. The total energy per unit volume $e$, is related to $p$ by the equation of state for a perfect gas

$$
p=(\gamma-1)\left[e-\frac{\left(m^{2}+n^{2}\right)}{2 \rho}\right]
$$

where $\gamma$ is the ratio of specific heats and should not be confused with the $\gamma_{j+1 / 2}$ in $(2.22 \mathrm{c})$ or $\gamma_{j+1 / 2}^{l}$ in $(3.7 \mathrm{~d})$. 
Let $A$ denote the Jacobian matrix $\partial F(U) / \partial U$ whose eigenvalues are

$$
\left(a_{x}^{1}, a_{x}^{2}, a_{x}^{3}, a_{x}^{4}\right)=(u-c, u, u+c, u)
$$

where $c$ is the local speed of sound. The right eigenvectors of $A$ form the matrix $R_{x}=\left(R_{x}^{1}, R_{x}^{2}, R_{x}^{3}, R_{x}^{4}\right)$ given by

$$
R_{x}=\left[\begin{array}{cccc}
1 & 1 & 1 & 0 \\
u-c & u & u+c & 0 \\
v & v & v & 1 \\
H-u c & \left(u^{2}+v^{2}\right) / 2 & H+u c & v
\end{array}\right]
$$

where

$$
H=\frac{c^{2}}{\gamma-1}+\frac{u^{2}+v^{2}}{2}
$$

and

$$
R_{x}^{-1}=\left[\begin{array}{cccc}
\frac{1}{2}\left(b_{1}+u / c\right) & \frac{1}{2}\left(-b_{2} u-1 / c\right) & \frac{1}{2}\left(-b_{2} v\right) & \frac{1}{2} b_{2} \\
1-b_{1} & b_{2} u & b_{2} v & -b_{2} \\
\frac{1}{2}\left(b_{1}-u / c\right) & \frac{1}{2}\left(-b_{2} u+1 / c\right) & \frac{1}{2}\left(-b_{2} v\right) & \frac{1}{2} b_{2} \\
-v & 0 & 1 & 0
\end{array}\right]
$$

with

$$
\begin{aligned}
& b_{1}=b_{2} \frac{\left(u^{2}+v^{2}\right)}{2} \\
& b_{2}=\frac{\gamma-1}{c^{2}}
\end{aligned}
$$

Let the grid spacing be denoted by $\Delta x$ and $\Delta y$ such that $x=j \Delta x$ and $y=k \Delta y$. Using the same notation as in section 4 , the vector $\alpha$ of equation (3.6) for the $x$-direction (omitting the $k$ index) is 


$$
\left[\begin{array}{c}
\alpha_{j+1 / 2}^{1} \\
\alpha_{j+1 / 2}^{2} \\
\alpha_{j+1 / 2}^{3} \\
\alpha_{j+1 / 2}^{4}
\end{array}\right]=\left[\begin{array}{c}
(a a-b b) / 2 \\
\Delta_{j+1 / 2} \rho-a a \\
(a a+b b) / 2 \\
\Delta_{j+1 / 2} n-v_{j+1 / 2} \Delta_{j+1 / 2} \rho
\end{array}\right]
$$

where

$$
\begin{array}{r}
a a=\frac{\gamma-1}{c_{j+1 / 2}^{2}}\left[\Delta_{j+1 / 2} e+\frac{u_{j+1 / 2}^{2}+v_{j+1 / 2}^{2}}{2} \Delta_{j+1 / 2} \rho\right. \\
\left.-u_{j+1 / 2} \Delta_{j+1 / 2} m-v_{j+1 / 2} \Delta_{j+1 / 2} n\right] \\
b b=\left[\Delta_{j+1 / 2} m-u_{j+1 / 2} \Delta_{j+1 / 2} \rho\right] / c_{j+1 / 2}
\end{array}
$$

Similarly, let B denote the Jacobian matrix $\partial G(U) / \partial U$ whose eigenvalues are

$$
\left(a_{y}^{1}, a_{y}^{2}, a_{y}^{3}, a_{y}^{4}\right)=(v-c, v, v+c, v)
$$

where $c$ is the local speed of sound. The right eigenvectors of $B$ form the matrix $R_{y}=\left(R_{y}^{1}, R_{y}^{2}, R_{y}^{3}, R_{y}^{4}\right)$ given by

$$
R_{y}=\left[\begin{array}{cccc}
1 & 1 & 1 & 0 \\
u & u & u & 1 \\
v-c & v & v+c & 0 \\
H-v c & \left(u^{2}+v^{2}\right) / 2 & H+v c & u
\end{array}\right]
$$

and

$$
R_{y}^{-1}=\left[\begin{array}{cccc}
\frac{1}{2}\left(b_{1}+v / c\right) & \frac{1}{2}\left(-b_{2} u\right) & \frac{1}{2}\left(-b_{2} v-1 / c\right) & \frac{1}{2} b_{2} \\
1-b_{1} & b_{2} u & b_{2} v & -b_{2} \\
\frac{1}{2}\left(b_{1}-v / c\right) & \frac{1}{2}\left(-b_{2} u\right) & \frac{1}{2}\left(-b_{2} v+1 / c\right) & \frac{1}{2} b_{2} \\
-u & 1 & 0 & 0
\end{array}\right]
$$


with $b_{1}$ and $b_{2}$ defined in equations (5.3d) and (5.3e). The vector $\alpha$ for the $y$-direction (omitting the $j$ index) is

$$
\left[\begin{array}{c}
\alpha_{k+1 / 2}^{1} \\
\alpha_{k+1 / 2}^{2} \\
\alpha_{k+1 / 2}^{3} \\
\alpha_{k+1 / 2}^{4}
\end{array}\right]=\left[\begin{array}{c}
(c c-d d) / 2 \\
\Delta_{k+1 / 2} \rho-c c \\
(c c+d d) / 2 \\
\Delta_{k+1 / 2} m-u_{k+1 / 2} \Delta_{k+1 / 2} \rho
\end{array}\right]
$$

where

$$
\begin{array}{r}
c c=\frac{\gamma-1}{c_{k+1 / 2}^{2}}\left[\Delta_{k+1 / 2} e+\frac{u_{k+1 / 2}^{2}+v_{k+1 / 2}^{2}}{2} \Delta_{k+1 / 2} \rho\right. \\
\left.-u_{k+1 / 2} \Delta_{k+1 / 2} m-v_{k+1 / 2} \Delta_{k+1 / 2} n\right] \\
d d=\left[\Delta_{k+1 / 2} n-v_{k+1 / 2} \Delta_{k+1 / 2} \rho\right] / c_{k+1 / 2}
\end{array}
$$

As mentioned previously, the simplest form for $U_{j+1 / 2, k}$ is

$$
U_{j+1 / 2, k}=\left(U_{j+1, k}+U_{j, k}\right) / 2
$$

Roe's special form of the averaging in the $x$-direction is: 


$$
\begin{aligned}
u_{j+1 / 2, k} & =\frac{\bar{D} u_{j+1, k}+u_{j, k}}{\bar{D}+1} \\
v_{j+1 / 2, k} & =\frac{\bar{D} v_{j+1, k}+v_{j, k}}{\bar{D}+1} \\
H_{j+1 / 2, k} & =\frac{\bar{D} H_{j+1, k}+H_{j, k}}{\bar{D}+1} \\
c_{j+1 / 2, k}^{2} & =(\gamma-1)\left[H_{j+1 / 2, k}-\frac{1}{2}\left(u_{j+1 / 2, k}^{2}+v_{j+1 / 2, k}^{2}\right)\right] \\
\bar{D} & =\sqrt{\rho_{j+1, k} / \rho_{j, k}} \\
H & =\frac{\gamma p}{(\gamma-1) \rho}+\frac{1}{2}\left(u^{2}+v^{2}\right)
\end{aligned}
$$

Therefore to use Roe's averaging for the $x$-differencing, all one has to do is compute $u_{j+1 / 2, k}, v_{j+1 / 2, k}$, and $c_{j+1 / 2, k}$ in (5.2)-(5.4) by (5.9). Similarly, we can obtain the Roe's averaging for $u_{j, k+1 / 2}, v_{j, k+1 / 2}$, and $c_{j, k+1 / 2}$. In the numerical experiments for the two-dimensional test problem, we used Roe's averaging.

The two-dimensional form of numerical fluxes (3.7b) for the Euler equations of gas dynamics (5.1) can be written as

$$
\begin{aligned}
\tilde{F}_{j+1 / 2, k}^{n} & =\frac{1}{2}\left[F\left(U_{j, k}\right)+F\left(U_{j+1, k}\right)\right] \\
& +\frac{1}{2} \sum_{l=1}^{4}\left[g_{j}^{l}+g_{j+1}^{l}-Q\left(a_{j+1 / 2}^{l}+\gamma_{j+1 / 2}^{l}\right) \alpha_{j+1 / 2}^{l}\right] R_{j+1 / 2}^{l} \\
\check{G}_{j, k+1 / 2}^{n} & =\frac{1}{2}\left[G\left(U_{j, k}\right)+G\left(U_{j, k+1}\right)\right] \\
& +\frac{1}{2} \sum_{l=1}^{4}\left[g_{k}^{l}+g_{k+1}^{l}-Q\left(a_{k+1 / 2}^{l}+\gamma_{k+1 / 2}^{l}\right) \alpha_{k+1 / 2}^{l}\right] R_{k+1 / 2}^{l}(5.1
\end{aligned}
$$


with $g_{j+1}^{l}$ and $g_{k+1}^{l}$ corresponding to (3.7c) evaluated at the appropriate "locally frozen" lth characteristic speed and Ith characteristic variable in the $x$ - and $y$-direction, and $Q(z)$ is defined in (2.17d). The $\alpha_{j+1 / 2}^{l}$ and $\alpha_{k+1 / 2}^{l}$ are defined in (5.4) and (5.7). Also, the following notations have been used

$$
\begin{aligned}
a_{j+1 / 2}^{l} & \equiv\left(a_{x}^{l}\right)_{j+1 / 2, k} \\
a_{k+1 / 2}^{l} & \equiv\left(a_{y}^{l}\right)_{j, k+1 / 2} \\
\gamma_{j+1 / 2}^{l} & \equiv\left(\gamma_{x}^{l}\right)_{j+1 / 2, k} \\
\gamma_{k+1 / 2}^{l} & \equiv\left(\gamma_{y}^{l}\right)_{j, k+1 / 2}
\end{aligned}
$$

where $a_{x}^{l}$ is defined in (5.2), $a_{y}^{l}$ is defined in (5.5) and

$$
\begin{array}{r}
\gamma_{j+1 / 2}^{l}= \begin{cases}\left(g_{j+1}^{l}-g_{j}^{l}\right) / \alpha_{j+1 / 2}^{l} & \alpha_{j+1 / 2}^{l} \neq 0 \\
0 & \alpha_{j+1 / 2}^{l}=0\end{cases} \\
\gamma_{k+1 / 2}^{l}= \begin{cases}\left(g_{k+1}^{l}-g_{k}^{l}\right) / \alpha_{k+1 / 2}^{l} & \alpha_{k+1 / 2}^{l} \neq 0 \\
0 & \alpha_{k+1 / 2}^{l}=0,\end{cases}
\end{array}
$$

Here, it is understood that the scalar values and the vector $R^{l}$ in the summation in equations (5.10a) and (5.10b) are values of (5.2)-(5.9) evaluated at the coresponding $x$ - and $y$-coordinates. For simplicity, we omitted the $k$ index inside the summation sign of equation (5.10a), and omitted the $j$ index inside the summation sign of equation (5.10b).

\section{§5.2 Extension of the Explicit TVD Scheme by the Fractional Step Method}

In this subsection, we are going to review the implementation of the explicit $T V D$ scheme to two dimensions by the fractional step (time splitting) method. We will also give a discussion on the use of the artificial compression term. Later, we will show a comparision between the explicit and implicit $T V D$ schemes. 
The explicit $T V D$ scheme can be implemented in two space dimensions by the method of fractional steps as follows:

$$
\begin{aligned}
U_{j, k}^{*} & =U_{j, k}^{n}-\frac{\Delta t}{\Delta x}\left(\tilde{F}_{j+1 / 2, k}^{n}-\tilde{F}_{j-1 / 2, k}^{n}\right)=L_{x} U_{j, k}^{n} \\
U_{j, k}^{n+1}=U_{j, k}^{*}-\frac{\Delta t}{\Delta y}\left(\tilde{G}_{j, k+1 / 2}^{*}-\tilde{G}_{j, k-1 / 2}^{*}\right) & =L_{y} U_{j, k}^{*}
\end{aligned}
$$

that is

$$
U_{j, k}^{n+1}=L_{y} L_{x} U_{j, k}^{n}
$$

where $\tilde{F}_{j+1 / 2, k}^{n}$ and $\tilde{G}_{j, k+1 / 2}^{n}$ are defined in (5.10).

In order to retain the original time accuracy of the method, we use a Strang type of fractional step operators, namely

$$
U_{j, k}^{n+2}=L_{x} L_{y} L_{y} L_{x} U_{j, k}^{n}
$$

or for steady-state calculations, we use

$$
U_{j, k}^{n+2}=L_{x}^{*} L_{y} L_{x} L_{y} \ldots L_{y} L_{x}^{*} U_{j, k}^{0}
$$

where $L_{x}^{*}$ denotes the operator with the time step equal to $\Delta t / 2$.

We may enhance resolution the same way as discussed in section 2.5 by increasing the value of $\gamma_{j+1 / 2}^{l}$ (and $\gamma_{k+1 / 2}^{l}$ ) in equation (5.10g) (and (5.10h)). To accomplish this, we increase the size of the corresponding $g_{j}^{l}$ in (3.7c), for example, by multiplying the right-hand-side of $(3.7 \mathrm{c})$ by $\left[1+\omega^{l} \theta_{j}^{l}\right]$; i.e.

$$
\tilde{g}_{j}^{l}=\left[1+\omega^{l} \theta_{j}^{l}\right] g_{j}^{l}, \quad \omega^{l}>0
$$

with 


$$
\theta_{j}^{l}=\frac{\left|\alpha_{j+1 / 2}^{l}-\alpha_{j-1 / 2}^{l}\right|}{\left|\alpha_{j+1 / 2}^{l}\right|+\left|\alpha_{j-1 / 2}^{l}\right|}
$$

where $\alpha_{j+1 / 2}^{l}$ is defined by (5.4a). Similarily, we can obtain $\tilde{g}_{k}^{l}=\left[1+\omega^{l} \theta_{k}^{l}\right] g_{k}^{l}$ for the $y$-direction.

A preliminary experiment in two-dimensional calculations using the explicit TVD scheme by the fractional step approach indicated aneed for such an enhancement mechanism [2]. The artificial compression term is especially needed for the linearly degenerate characteristic field, i.e., $a_{x}=u$ and $a_{y}=$ $v$. We will use this form of the $\tilde{g}$ function for our two-dimensional numerical experiments.

\$5.3 Extension of the Implicit Scheme by the Alternating Direction Implicit (ADI) Method

The two-dimensional linearized nonconservative implicit (LNI) form of (3.8) for the Euler equations of gas dynamics (5.1) can be written as

$$
\begin{gathered}
{\left[I-\lambda^{x} J_{j+1 / 2, k}^{-} \Delta_{j+1 / 2, k}+\lambda^{x} J_{j-1 / 2, k}^{+} \Delta_{j-1 / 2, k}\right.} \\
\left.-\lambda^{y} K_{j, k+1 / 2} \Delta_{j, k+1 / 2}+\lambda^{y} K_{j, k-1 / 2}^{+} \Delta_{j, k-1 / 2}\right]\left(U^{n+1}-U^{n}\right) \\
=-\lambda^{x}\left[\tilde{F}_{j+1 / 2, k}^{n}-\tilde{F}_{j-1 / 2, k}^{n}\right]-\lambda^{y}\left[\tilde{G}_{j, k+1 / 2}^{n}-\tilde{G}_{j, k-1 / 2}^{n}\right]
\end{gathered}
$$

with $\lambda^{x}=\Delta t / \Delta x, \lambda^{y}=\Delta t / \Delta y$, where

$$
\begin{aligned}
J_{j+1 / 2, k}^{ \pm} & =\left(R_{x} \operatorname{diag}\left(C_{x}^{ \pm}\right) R_{x}^{-1}\right)_{j+1 / 2, k}^{n} \\
K_{j, k+1 / 2}^{ \pm} & =\left(R_{y} \operatorname{diag}\left(C_{y}^{ \pm}\right) R_{y}^{-1}\right)_{j, k+1 / 2}^{n}
\end{aligned}
$$

and 


$$
\begin{gathered}
\left(C_{x}^{ \pm}\right)_{j+1 / 2, k}^{n}=\frac{1}{2}\left[Q\left(a_{x}^{l}+\gamma_{x}^{l}\right) \pm\left(a_{x}^{l}+\gamma_{x}^{l}\right)\right]_{j+1 / 2, k}^{n} \\
\left(C_{y}^{ \pm}\right)_{j, k+1 / 2}^{n}=\frac{1}{2}\left[Q\left(a_{y}^{l}+\gamma_{y}^{l}\right) \pm\left(a_{y}^{l}+\gamma_{y}^{l}\right)\right]_{j, k+1 / 2}^{n} \\
l=1,2,3,4
\end{gathered}
$$

It is well known that solving the two-dimensional implicit difference equation (5.16) is very costly. This leads to the popularity of using the alternating direction implicit $(A D I)$ method to solve gas dynamics problems. Formally, we can write an $A D I$ form of (5.16) as

$$
\begin{aligned}
{\left[I-\lambda^{x} J_{j+1 / 2, k}^{-} \Delta_{j+1 / 2, k}+\lambda^{x} J_{j-1 / 2, k}^{+} \Delta_{j-1 / 2, k}\right] D^{*} } & = \\
-\lambda^{x}\left[\tilde{F}_{j+1 / 2, k}^{n}-\tilde{F}_{j-1 / 2, k}^{n}\right]-\lambda^{y}\left[\tilde{G}_{j, k+1 / 2}^{n}-\tilde{G}_{j, k-1 / 2}^{n}\right] & \\
{\left[I-\lambda^{y} K_{j, k+1 / 2}^{-} \Delta_{j, k+1 / 2}+\lambda^{y} K_{j, k-1 / 2}^{+} \Delta_{j, k-1 / 2}\right] D } & =D^{*} \\
U^{n+1} & =U^{n}+D
\end{aligned}
$$

Given $U_{j, k}^{n}$, for each $(j, k)$, we now list the operations needed to calculate $U_{j, k}^{n+1}$ by the $A D I$ method (assuming a given $C F L$ number):

(i) compute $u_{j, k}=m_{j, k} / \rho_{j, k}, v_{j, k}=n_{j, k} / \rho_{j, k}$ and $p_{j, k}$

(ii) compute $u_{j+1 / 2, k}, v_{j+1 / 2, k}$ and $c_{j+1 / 2, k}$ from (5.8) or (5.9), calculate

$$
\begin{aligned}
& M_{x}=\max _{j, k}\left(\left|u_{j+1 / 2, k}\right|+c_{j+1 / 2, k}\right) \\
& M_{y}=\max _{j, k}\left(\left|v_{j+1 / 2, k}\right|+c_{j+1 / 2, k}\right)
\end{aligned}
$$

evaluate $\alpha_{j+1 / 2}^{l}, l=1,2,3,4$ by (5.4a), (remember that the $k$ index is omitted from the equation), and define 


$$
\lambda^{x}=\Delta t / \Delta x=\mu / \max _{j, k}\left(M_{x}, M_{y}\right)
$$

where $\mu$ is the prescribed CFL as input

(iii) compute $\left(a_{x}^{l}\right)_{j+1 / 2, k}$ by (5.2), $g_{j+1 / 2, k}^{l}$ by (3.7c), (with $\left(a_{x}^{l}\right)_{j+1 / 2, k}$ as $a_{j+1 / 2}^{l}$ in $\left.(3.7 \mathrm{c})\right)$

(iv) compute $\left(\gamma_{x}^{l}\right)_{j+1 / 2, k}$ by $(5.10 \mathrm{~g})$, and $\tilde{F}_{j+1 / 2, k}$ by $(5.10 \mathrm{a})$ and relation (5.3a)

(v) compute $C_{\frac{x}{x}}^{ \pm}$by (5.16d) (with $z=\left(a_{x}^{l}\right)_{j+1 / 2, k}+\left(\gamma_{x}^{l}\right)_{j+1 / 2, k}$ in equation (2.18a))

(vi) compute $J_{j+1 / 2, k}^{ \pm}$by $(5.16 \mathrm{~b})$

(vii) compute $u_{j, k+1 / 2}, v_{j, k+1 / 2}$ and $c_{j, k+1 / 2}$, calculate

$$
\begin{aligned}
& M_{x}=\max _{j, k}\left(\left|u_{j, k+1 / 2}\right|+c_{j, k+1 / 2}\right) \\
& M_{y}=\max _{j, k}\left(\left|v_{j, k+1 / 2}\right|+c_{j, k+1 / 2}\right)
\end{aligned}
$$

evaluate $\alpha_{k+1 / 2}^{l}, l=1,2,3,4$ by (5.7a) (remember that the $j$ index is omitted from the equation) and define

$$
\lambda^{y}=\Delta t / \Delta y=\mu / \max _{j, k}\left(M_{x}, M_{y}\right)
$$

(ix) compute $\left(a_{y}^{l}\right)_{j, k+1 / 2}$ by (5.5), $g_{j, k+1 / 2}^{l}$ by (3.7c), (with $\left(a_{x}^{l}\right)_{j, k+1 / 2}$ as $a_{k+1 / 2}^{l}$ in (3.7c))

(x) compute $\left(\gamma_{y}^{l}\right)_{j, k+1 / 2}$ by $(5.10 \mathrm{~h})$, and $\tilde{G}_{j, k+1 / 2}$ by $(5.10 \mathrm{~b})$ and relation (5.6a)

(xi) solve equation (5.17a) for $D^{*}$.

(xii) compute $C_{y}^{\frac{1}{y}}$ by $\left(5.16 \mathrm{e}\right.$ ) (with $z=\left(a_{y}^{l}\right)_{j, k+1 / 2}+\left(\gamma_{y}^{l}\right)_{j, k+1 / 2}$ in equation (2.18a)) 
(xiii) compute $K_{j, k+1 / 2}^{ \pm}$by (5.16c)

(xiv) solve equation (5.17c) for $U_{j, k}^{n+1}$.

We now turn to the discussion of the influence of the approximate factorization error on rate of convergence for the $A D I$ method. It is well known that contrary to the fact that the convergence rate for the unfactored scheme is directly proportional to the CFL number, the convergent rate for a factored scheme has a definite maximum. That is, the iteration count grows rapidly when the calculation is carried out away from an optimal time-step. Recently, Abarbanel et. al. $[22,23]$ have made some detailed analyses of the influence of the approximate factorization error on the efficiency of convergence rate as a function of CFL number for a conventional $A D I$ scheme. We feel that, in our case, this phenomenon is related to the fact that the $A D I$ version ceased to be $T V D$ (in the scalar case) for large enough $C F L$ number. At this time, however, we do not have a rigorous analysis for the dependence of the rate of convergence on the $C F L$ number. Here, we extend (5.16) to an $A D I$ form for the purpose of numerical experiments.

\section{§5.4 Numerical Example}

In order to examine the applicability of the new method for twodimensional shock calculations, we consider a simple inviscid flow field developed by a shock wave reflecting from a rigid surface (figure(5.1)). The steady-state solution can be calculated exactly and thus can aid us in evaluating the quality of the numerical method. Figure (5.1) shows the indexing of the computational mesh.

\section{Initial Conditions:}

Initially, the entire flow field is set equal to the freestream supersonic inflow values plus the analytical boundary conditions as described below.

\section{Analytical Boundary Conditions:}

The boundary conditions are given as follows: (a) supersonic inflow at $j=1, k=1, \ldots, K$ which allows the values $U_{1, k}$ to be fixed at freestream conditions; (b) prescribed fixed values of $U_{j, k}$ at $k=K, j=1, \ldots, J$ which produce the desired shock strength and shock angle; (c) supersonic outflow at $j=J, k=1, \ldots, K$; (d) a rigid flat surface at $k=1, j=1, \ldots, J$ which can be shown to be properly represented by the condition $v=0$, with the additional condition $\partial p / \partial y=0$ at $k=1$ from the normal $y$-momentum 
equation.

Numerical Boundary Conditions:

The supersonic outflow values $U_{J, k}, k=1, \ldots, K-1$ are obtained by zeroth-order extrapolation, i.e.,

$$
U_{J, k}=U_{J-1, k}, \quad k=1, \ldots, K-1
$$

The values of $\rho_{j, 1}, m_{j, 1}$ on the rigid surface with $j=1, \ldots, J$ are also obtained by zeroth-order extrapolation, i.e.,

$$
\begin{aligned}
\rho_{j, 1} & =\rho_{j, 2} \\
m_{j, 1} & =m_{j, 2}
\end{aligned} \quad j=1, \ldots, J
$$

Three different methods were used in approximating $\partial p / \partial y=0$ to get $e_{j, 1}, j=1, \ldots, J$

(a) normal derivative of $e$ equal to zero (first-order)

$$
e_{j, 1}=e_{j, 2}
$$

(b) normal derivative of $e$ equal to zero (second-order)

$$
e_{j, 1}=\left(4 e_{j, 2}-e_{j, 3}\right) / 3
$$

(c) normal derivative of $p$ equal to zero (second-order)

$$
\begin{aligned}
& p_{j, 1}=\left(4 p_{j, 2}-p_{j, 3}\right) / 3 \\
& e_{j, 1}=\frac{p_{j, 1}}{(\gamma-1)}+\frac{m_{j, 1}^{2}}{2 \rho_{j, 1}}
\end{aligned}
$$

Equation (5.20a) together with equation (5.19) is an approximation to $p_{j, 1}=p_{j, 2}$; i.e., a first-order approximation for the normal derivative of 
$p$ equal to zero. Equation (5.20b) together with equation (5.19) is an approximation to equation $(5.20 \mathrm{c})$. We use equations $(5.20 \mathrm{a})$ or $(5.20 \mathrm{~b})$ for the implicit method mainly because of their ease of application with implicit numerical boundary conditions. From the numerical experiments, we found that equation $(5.20 \mathrm{~b})$ and $(5.20 \mathrm{c})$ produce better numerical solutions near the wall than equation (5.20a).

Since this implicit TVD scheme is a 5-point scheme (in each spatial direction), we also need the values of $g_{1, k}, g_{J, k}, g_{j, 1}, g_{j, K}, \theta_{1, k}, \theta_{J, k}, \theta_{j, 1}, \theta_{j, K}$, for $j=1, \ldots, J$ or $k=1, \ldots, K$. For convenience, we will set

$$
\begin{aligned}
g_{1, k} & =g_{2, k} & \theta_{1, k} & =\theta_{2, k} \\
g_{J, k} & =g_{J-1, k} & \theta_{J, k} & =\theta_{J-1, k} \\
g_{j, 1} & =g_{j, 2} & \theta_{j, 1} & =\theta_{j, 2} \\
g_{j, K} & =g_{j, K-1} & \theta_{j, K} & =\theta_{j, K-1}
\end{aligned}
$$

\section{§5.5 Discussion of Numerical Results}

The purpose of these numerical experiments is three fold:

(i) To test the performance of the $A D I$ form of the implicit TVD scheme (5.17) on the shock reflection problem. For reference purposes, we denote scheme (5.17) as STVD.

(ii) To test the performance of scheme (5.17) with $\gamma_{x}^{l}=\gamma_{y}^{l}=0$ in (5.16b) and (5.16c) (i.e., first-order spatial difference for the implicit operator) on the shock reflection problem. For reference purposes, we denote this method as FTVD.

(iii) To test the effect of the choice of $\sigma$ and $Q$ function in (2.22), and the artificial compression term in (5.15) on the convergence rate and resolution of the above two-dimensional problem.

In all of the numerical experiments, the incident shock angle $\psi$ was $29^{\circ}$ and the freestream Mach number $M_{\infty}$ was 2.9. The computational domain was $0 \leq x \leq 4.1$, and $0 \leq y \leq 1$, with a uniform grid size of $61 \times 21$. The appropriate analytical boundary conditions were applied along the boundaries of the domain. Only pressure contours and pressure coefficients will be illustrated. Here, the pressure coefficient is defined as $c_{p}=\frac{2}{\gamma M_{\infty}^{2}}\left(p / p_{\infty}-1\right)$ 
with $\gamma$ the ratio of specific heats, and $p_{\infty}$ the freestream pressure. The exact minimum pressure corresponding to $\psi=29^{\circ}$ and $M_{\infty}=2.9$ is 0.714286 and the exact maximum pressure is 2.93398 . The exact pressure solution and the computation domain is shown in figure (5.2). Forty-one pressure contour levels between the values of 0 and 4 with uniform increment 0.1 were used for the contour plots. The pressure coefficient was evaluated at $y=0.5$ for $0 \leq$ $x \leq 4.1$. All of the computations for the two-dimensional shock reflection problem were done in single precision on a CDC 7600 computer.

Comparison of Method

Pressure contours and the pressure coefficients evaluated at $y=0.5$ are shown in figure (5.3) for four different methods. Figure (5.3a) shows the numerical result of the explicit $T V D$ scheme by the fractional step method (5.14) with

$$
\begin{aligned}
& Q(z)=z^{2}+1 / 4 \\
& \sigma(z)=1 / 8
\end{aligned}
$$

It took approximately 350 steps to converge with a fixed $C F L=0.8$. The average smearing of the shocks is two points. Figure (5.3b) shows the steadystate solution of the conventional implicit method [21] with $C F L=1.0$ after approximately 600 steps. The oscillations are spread over 8 grid points. The experimental maximum $C F L$ number for this conventional implicit method is around 1.5. Figure (5.3c) shows the numerical result of the $A D I$ form of the implicit TVD scheme (5.17) ( $S T V D$ method with $\delta=0.125$ and $\sigma$ in equation (2.22e) ) after 300 steps with $C F L=3$. The experimental maximum $C F L$ number for this method is 5 under the $C F L$ sampling sequence of $(1,2,3,5,10,20)$. Figure (5.3d) shows the numerical result of the FTVD method (with $\delta=0.125$ and $\sigma$ in equation (2.22e) ) after 60 steps with $C F L=6$. The average smearing of the shocks is 2 points. The convergence rate of the FTVD method is far better than the $S T V D$ method. It was found that the optimal $C F L$ (with $\Delta t$ fixed) for fastest convergence rate with the FTVD method is between 5 and 10 .

In order to show that the convergence rate is not a monotone decreasing function of $C F L$ number for the FTVD method, figure (5.4a) illustrates the same method as figure (5.3d) after 250 steps with $C F L=50$. The solution is not quite convergent yet. Figure $(5.4 \mathrm{~b})$ shows the same method after 250 steps with $C F L=500$ (We ran for another 400 steps, without reaching 
steady-state). Figure (5.4c) shows the same method after 250 steps with $C F L$ $=1000$.

A primary factor affecting the stability and convergence rate of the $A D I$ form of the implicit TVD scheme is the approximate factorization error. This will be a subject of further investigation.

The Choice of $\sigma$ and $Q$ Functions, and the Artificial Compression Term

The primary difference in shock resolution between the explicit and implicit $T V D$ methods of figures (5.3a) and (5.3c) is the result of using a different $\sigma$ and $Q$. Equations (2.22e) and (2.17d) are used for the implicit scheme and equation (5.22) is used for the explicit scheme. We have found that the shock resolution is somewhat degraded by the explicit TVD scheme if $\sigma$ in (2.22e) is used instead of (5.22). The particular choice of $\sigma$ in (2.22e) is especially appropriate for the $A D I$ method since the steady-state solutions are independent of $\Delta t$.

All of the TVD schemes were operators with an artificial compression term (5.15a) with $\omega^{l}=2, l=1,2,3,4$ except for the FTVD method. For the FTVD method, we used $\omega^{1}=\omega^{3}=1$ and $\omega^{2}=\omega^{4}=2$. We found that with this set of $\omega^{l}$, we get a faster convergence rate.

Approximate CPU Time and Actual Implementation of the Numerical Flux Functions

The conventional implicit method requires approximately twice the $C P U$ time per time step as the explicit TVD scheme. The implicit TVD method requires approximately 2.5 times more $C P U$ time per time step than the conventional implicit method.

In actual implementation of the explicit and implicit TVD methods into a computer code, the following form of the numerical flux $\tilde{F}_{j+1 / 2, k}^{n}$ (similarily for $\tilde{G}_{j, k+1 / 2}^{n}$ ) was used instead of (5.10)

$$
\begin{aligned}
& \tilde{F}_{j+1 / 2, k}^{n}=\frac{1}{2}\left[F\left(U_{j, k}\right)+F\left(U_{j+1, k}\right)\right] \\
& +\frac{1}{2} \sum_{l=1}^{4}\left[\xi_{j+1 / 2}^{l} \sigma_{j+1 / 2}^{l}\left(\vec{g}_{j}^{l}+\bar{g}_{j+1}^{l}\right)-Q\left(a_{j+1 / 2}^{l}+\gamma_{j+1 / 2}^{l}\right) \alpha_{j+1 / 2}^{l}\right] R_{j+1 / 2}^{l}
\end{aligned}
$$


with

$$
\xi_{j+1 / 2}^{l}=1+\omega^{l} \max \left(\theta_{j}^{l}, \theta_{j+1}^{l}\right)
$$

where $\theta_{j}^{l}$ is defined in equation $(5.15 \mathrm{~b})$, and

$$
\begin{aligned}
\bar{g}_{j}^{l} & =S \cdot \max \left[0, \min \left(\left|\alpha_{j+1 / 2}^{l}\right|, S \cdot \alpha_{j-1 / 2}^{l}\right)\right] \\
S & =\operatorname{sign}\left(\alpha_{j+1 / 2}^{l}\right)
\end{aligned}
$$

and

$$
\gamma_{j+1 / 2}^{l}=\xi_{j+1 / 2}^{l} \sigma_{j+1 / 2}^{l} \begin{cases}\left(\bar{g}_{j+1}^{l}-\bar{g}_{j}^{l}\right) / \alpha_{j+1 / 2}^{l} & \alpha_{j+1 / 2}^{l} \neq 0 \\ 0 & \alpha_{j+1 / 2}^{l}=0\end{cases}
$$

That is, the $\sigma_{j+1 / 2}^{l}$ has been taken out from the definition of $g_{j}^{l}$ in equation (3.7c) for simplicity. Furthermore, the artificial compression is incorporated into the definition of the numerical flux function.

\section{§6. Concluding Remarks}

The nonlinear, spatially second-order accurate, unconditionally stable implicit TVD scheme in a "linearized nonconservative" form has been applied to obtain steady-state solutions for the one-dimensional compressible inviscid equations of gas dynamics. This linearized form of the implicit TVD scheme is only conservative after the solution reaches steady-state. Numerical experiments for a quasi-one-dimensional nozzle problem show that the experimentally determined stability limit correlates exactly with the theoretical stability limit for the nonlinear scalar hyperbolic conservation laws. Steadystate solution can be reached in approximately 25 steps.

We have formally extended the second-order accurate implicit $T V D$ scheme by an $A D I$ method for two-dimensional calculations. Numerical experiments with the $A D I$ form (5.17) for a shock reflection problem show the gain in efficiency is not as pronounced as the one-dimensional counter part. 
A spatially first-order accurate left-hand side (i.e., by setting $\gamma_{x}^{l}=\gamma_{y}^{l}=0$ in (5.11b) and (5.11c)) of the $A D I$ form provides better stability and a faster convergence rate. A steady-state solution can be reached in approximately 60 steps for a two-dimensional shock reflection problem. Numerical experiments also show that the rate of convergence is very sensitive to the $C F L$ number. The iteration count grows rapidly when the calculation is carried out away from an optimal time-step.

More rigorous analysis of the influence of approximate factorization error on the stability and efficiency of the method is needed. This will be the subject of a future investigation.

\section{REFERENCES}

[1] R.D. Richtmyer and K.W. Morton, Difference Methods for Initial-Value Problems, Interscience-Wiley, New York, 1967.

[2] A. Harten, "A High Resolution Scheme for the Computation of Weak Solutions of Hyperbolic Conservation Laws, ${ }^{,}$NYU Report, March 1982.

[3] A. Harten, "On a Class of High Resolution Total-Variation-Stable Finite-Difference Schemes," NYU Report, 1982.

[4] A. Harten, J.M. Hyman and P.D. Lax, "On Finite-Difference Approximations and Entropy Conditions for Shocks," Comm. Pure Appl. Math., Vol. 29, 1976, pp. 297-322.

[5] A.Y. LeRoux, "A Numerical Conception of Entropy for Quasi-Linear Equations,". Math. Comp. Vol 31, No. 140, Oct. 1977, pp 848-872.

[6] M.G. Crandall and A. Majda, "Monotone Difference Approximations for Scalar Conservation Laws," Math. Comp. Vol 34, No 149, Jan. 1980, pp $1-21$.

[7] B. van Leer, "Towards the Ultimate Conservative Difference Scheme. V. A Second-Order Sequel to Godunov's Method," J. Comp. Phys., Vol. 32, 1979, pp. 101-136.

[8] P.K. Sweby and M.J. Baines, "Convergence of Roe's Scheme for the General Non-linear Scalar Wave Equation," Numerical Analysis Report 8/81, University of Reading, 1981.

[9] S. Osher and S. Chakravarthy, "High Resolution Schemes and the Entropy Condition," in preparation. 
[10] A. Harten and J.M. Hyman, "A Self-Adjusting Grid for the Computation of Weak Solutions of Hyperbolic Conservation Laws," Los Alamos Nat. Lab. Report LA9105, 1981.

[11] H.C. Yee, R.F. Warming and A. Harten, "On the Application and Extension of Harten's High-Resolution Scheme," NASA TM-84256, June 1982.

[12] H.C. Yee, R.F. Warming and A. Harten, "A High-Resolution Numerical Technique for Inviscid Gas-Dynamic Problems with Weak Solutions," Proc. Eighth International Conference on Numerical Methods in Fluid Dynamics, Aachen, West Germany, June 1982, Springer-Verlag.

[13] J. Glimm and P.D. Lax, "Decay of Solutions of Systems of Nonlinear Hyperbolic Conservation Laws," Mem. Amer. Math. Soc. 101, 1970.

[14] P.D. Lax, "Hyperbolic Systems of Conservation Laws and the Mathematical Theory of Shock Waves," SIAM, Philadelphia, 1972.

[15] P. Colella and P.R. Woodward, "The Piecewise-Parabolic Method (PPM) for Gas-Dynamical Simulations," LBL report no. 14661, July 1982.

[16] A. Harten, "The Artificial Compression Method for Computation of Shocks and Contact Discontinuities. I. Single Conservation Laws," Comm. Pure Appl. Math., Vol. XXX, 1977, pp. 611-638.

[17] A. Harten, "The Artificial Compression Method for Computation of Shocks and Contact Discontinuities: III. Self-Adjusting Hybrid Schemes," Math. Comp., Vol. 32, No. 142, 1978, pp. 363-389.

[18] P.L. Roe, "Approximate Riemann Solvers, Parameter Vectors, and Difference Schemes," J. Comp. Phys. Vol. 43, 1981, pp. 357-372.

[19] G.R. Shubin, A.B. Stephens, H.M. Glaz, "Steady Shock Tracking and Newton's Method Applied to One-Dimensional Duct Flow," J. Comp. Phys., Vol. 39, 1981, pp 364-374.

[20] J. Steger and R.F. Warming, "Flux Vector Splitting of the Inviscid Gas-dynamic Equations with Application to Finite Difference Methods," J. Comp. Phys., Vol. 40, No. 2, Apr. 1981, pp. 263-293.

[21] R.F. Warming and R.M. Beam, "On the Construction and Application of Implicit Factored Schemes for Conservation Laws," SIAMAMS Proceedings, Vol. 11, Symposium on Comp. Fluid Dynamics, New York, Apr. 1977.

[22] S.S. Abarbanel, D.L. Dwoyer and D. Gottlieb, "Improving the Convergence Rate of Parabolic ADI Method," ICASE Report No. 82-28, 
Sept. 1982.

[23] S.S. Abarbanel, D.L. Dwoyer and D. Gottlieb, "Stable Implicit Finite Difference Methods for Three-Dimensional Hyperbolic Systems," ICASE Report, (to be published, 1982). 


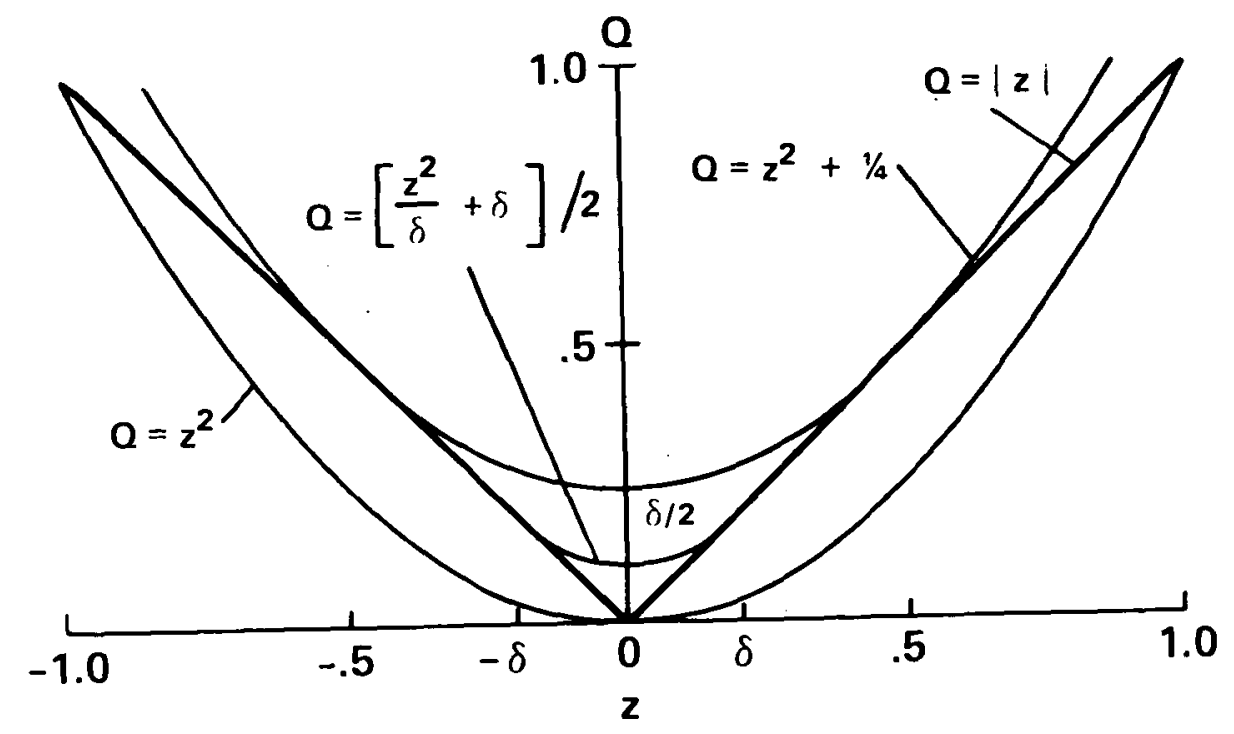

Fig. 2.1 Sample of the $Q(z)$ functions. 


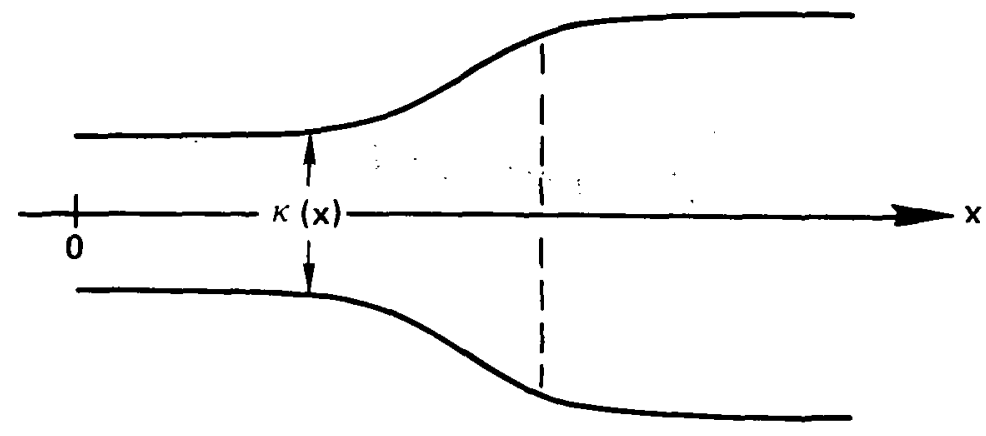

$\kappa(x)=1.398+0.347 * \tanh (0.8 x-4)$

Fig. 4.1 Divergent nozzle. 


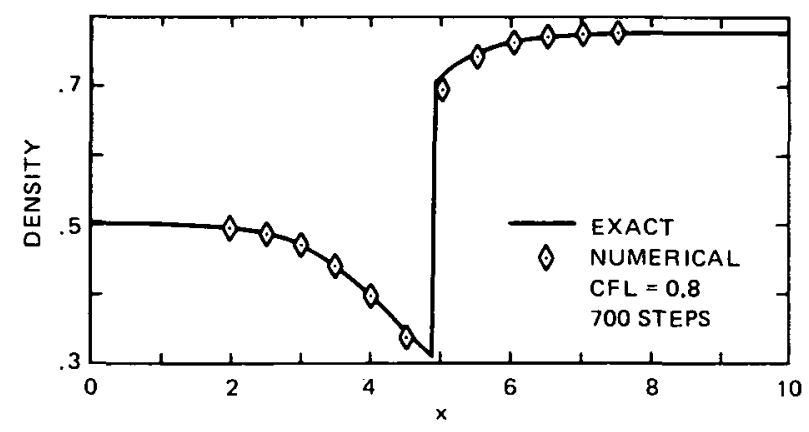

(a) Explicit $T V D$ method.

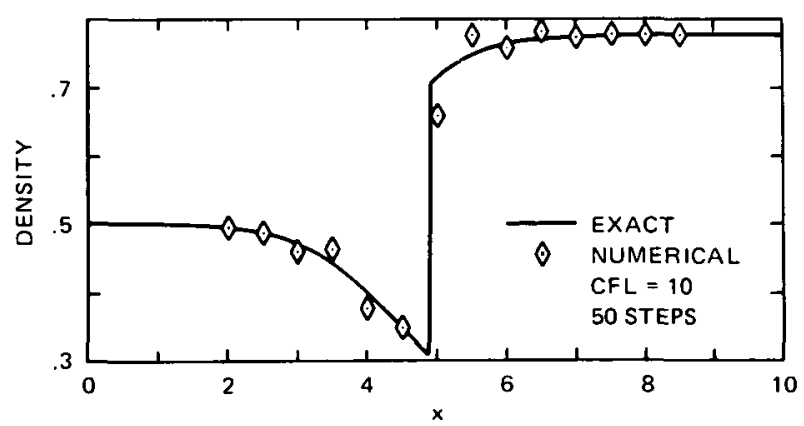

(c) Conventional implicit method.

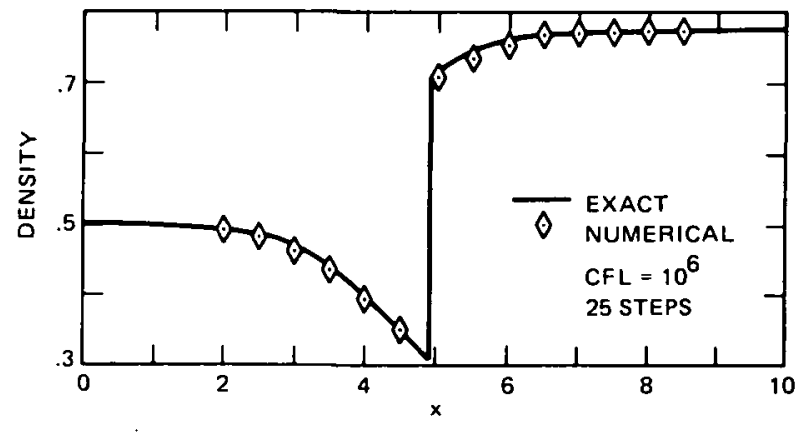

(b) Implicit $T V D$ method.

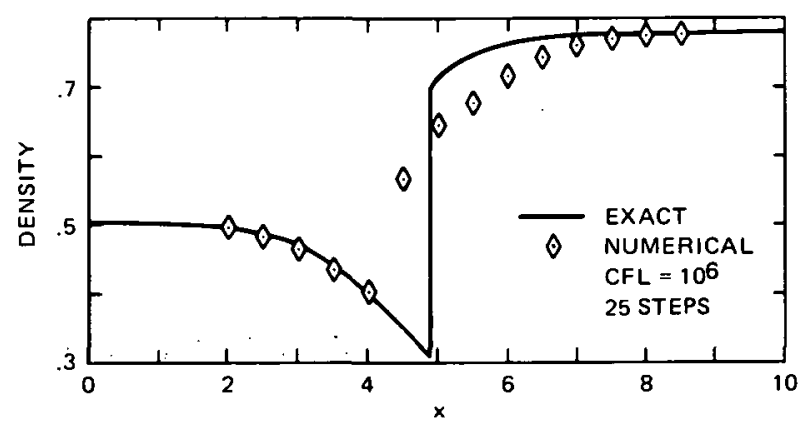

(d) First-order implicit flux-vector splitting method.

Fig. 4.2 Density distribution: supersonic inflow, subsonic outflow; 20 spatial intervals. 


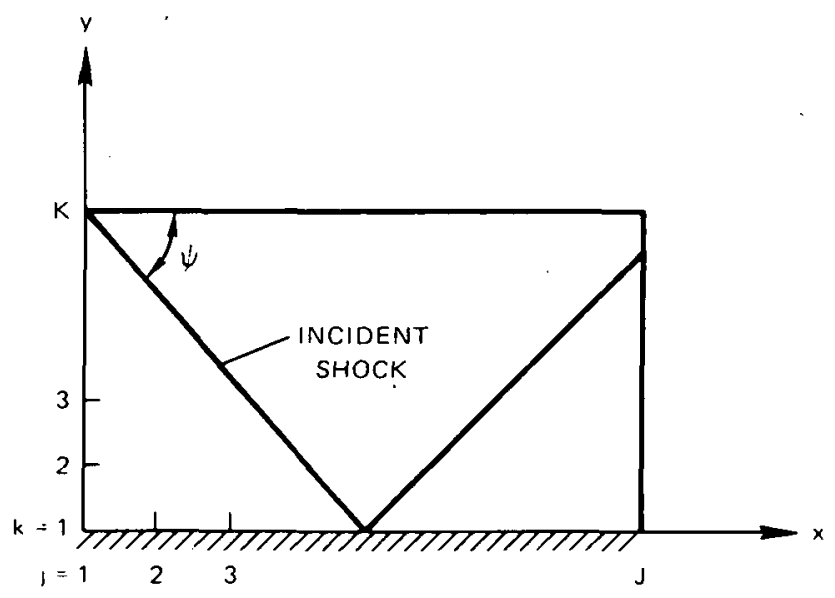

Fig. 5.1 Indexing of computational mesh for shock reflection problem.

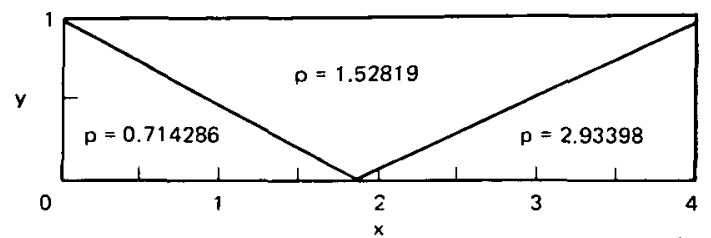

Fig. 5.2 The exact pressure solution for the shock reflection problem. 
PRESSURE CONTOURS

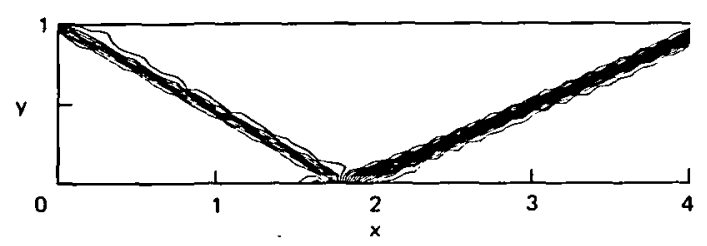

PRESSURE COEFFICIENTS

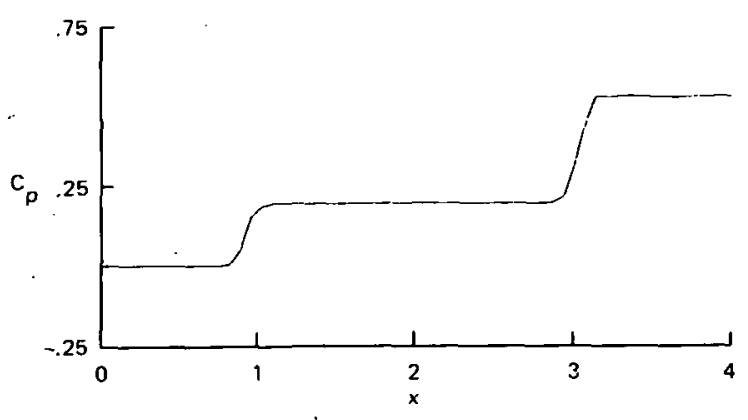

(a) Explicit TVD method, 350 steps, $C F L=0.8$.
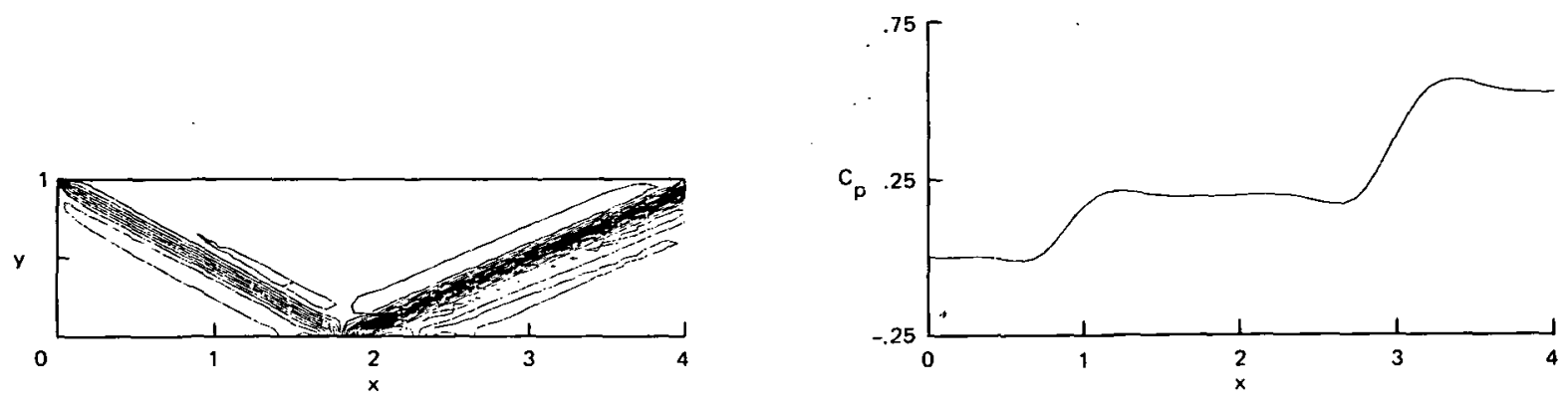

(b) Conventional implicit method, 600 steps, $C F L=1$.
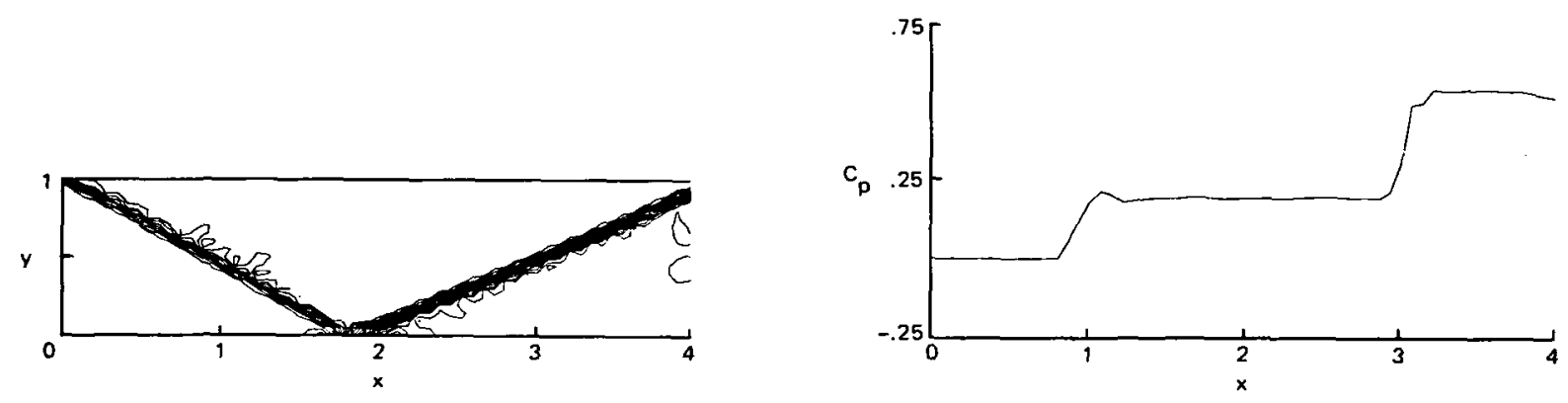

(c) Implicit $T V D$ method ( $S T V D$ ), 300 steps, $C F L=3$.
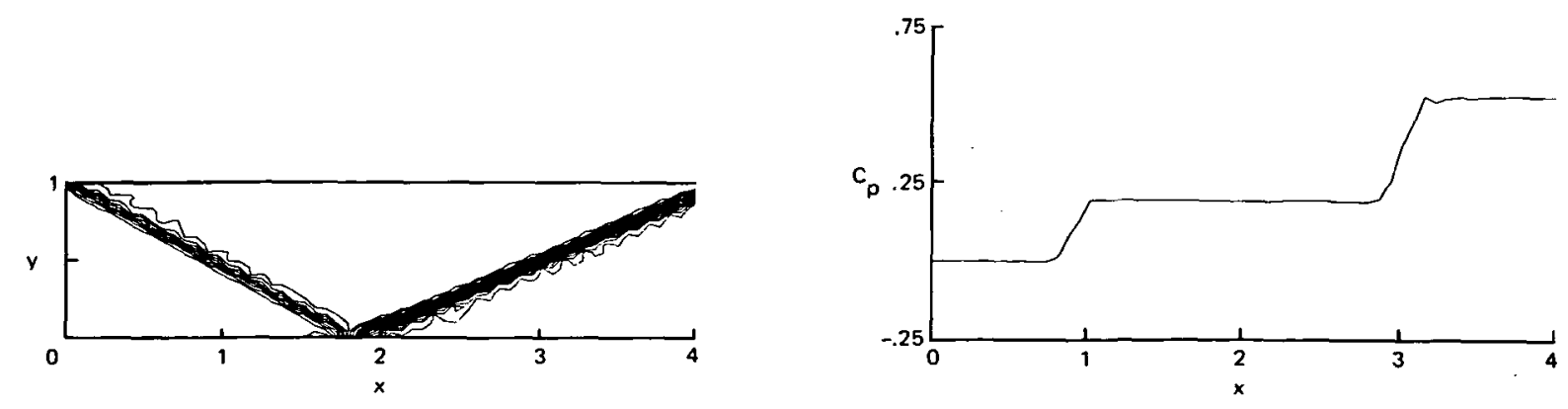

(d) Implicit $T V D$ method ( $F T V D$ ), 60 steps, $C F L=6$.

Fig. 5.3 Pressure contours and pressure coefficients-for-the shock reflection problem. 


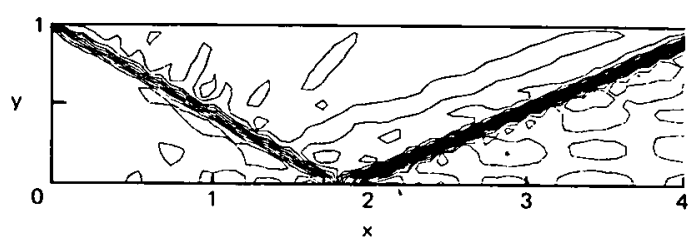

(a) 250 steps, $C F L=50$.

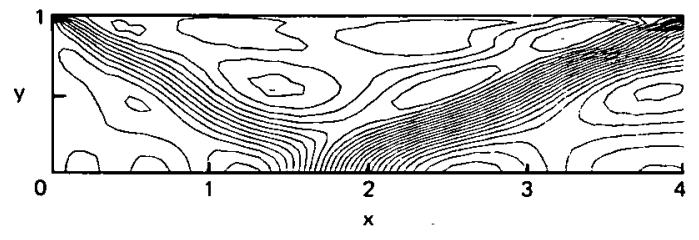

(b) 250 steps, $C F L=500$.

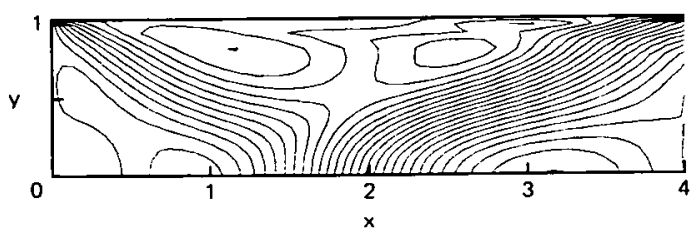

(c) 250 steps, $C F L=1000$.

Fig. 5.4 Pressure Contours for three different $C F L$ numbers. 


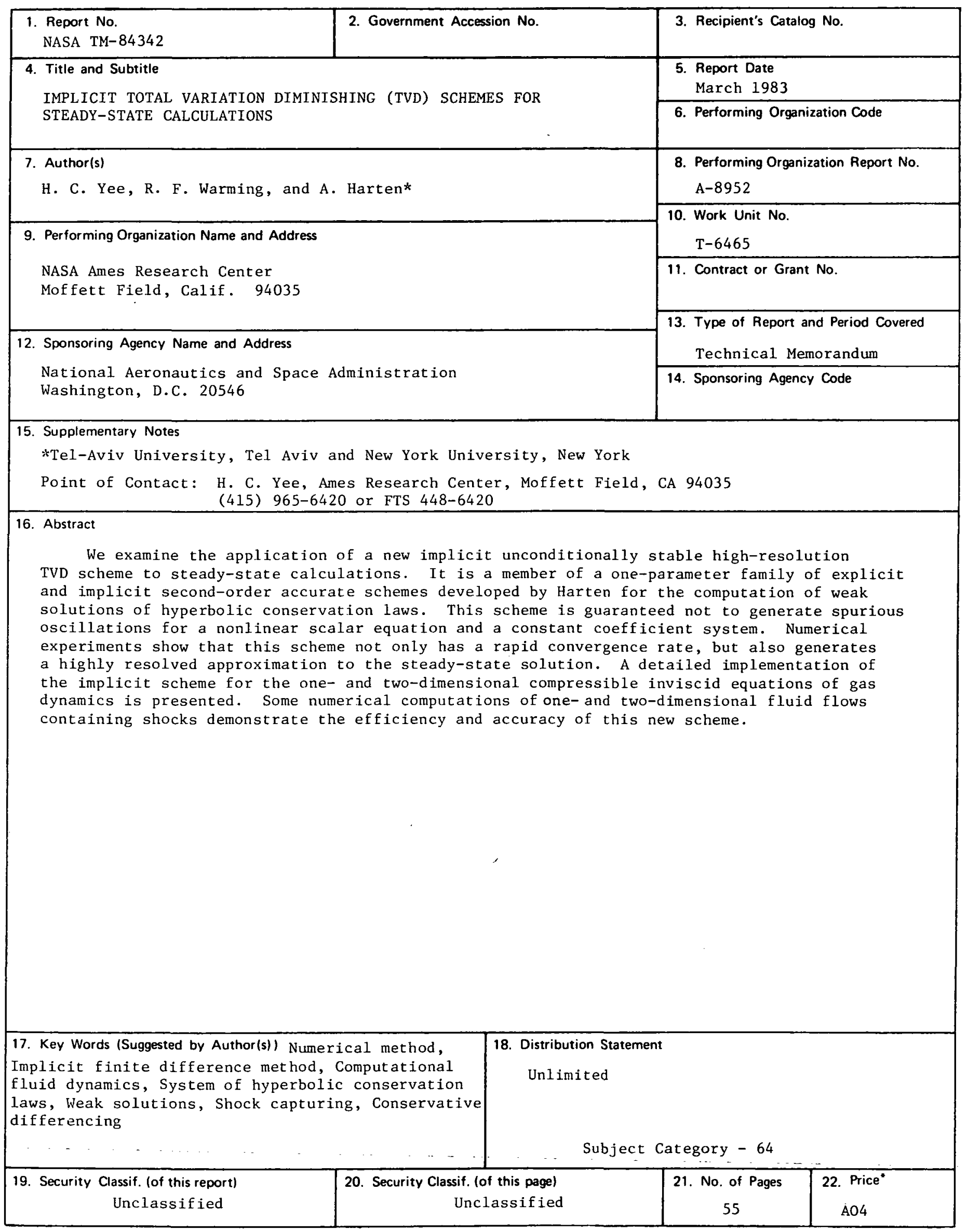

